\title{
On the Solution of NBVP for Multidimensional Hyperbolic Equations
}

\author{
Allaberen Ashyralyev and Necmettin Aggez \\ Department of Mathematics, Fatih University, Buyukcekmece, 34500 Istanbul, Turkey \\ Correspondence should be addressed to Necmettin Aggez; naggez@fatih.edu.tr
}

Received 16 August 2013; Accepted 10 February 2014; Published 25 May 2014

Academic Editors: A. Ibeas, L. Kong, and F. Mukhamedov

Copyright ( 2014 A. Ashyralyev and N. Aggez. This is an open access article distributed under the Creative Commons Attribution License, which permits unrestricted use, distribution, and reproduction in any medium, provided the original work is properly cited.

We are interested in studying multidimensional hyperbolic equations with nonlocal integral and Neumann or nonclassical conditions. For the approximate solution of this problem first and second order of accuracy difference schemes are presented. Stability estimates for the solution of these difference schemes are established. Some numerical examples illustrating applicability of these methods to hyperbolic problems are given.

\section{Introduction}

In the last decades, for the development of numerical methods and theory of solutions of the hyperbolic problems with nonlocal integral, Neumann and nonclassical conditions have been an important research topic in many natural phenomena. Solutions of this type of hyperbolic problems were investigated in [1-13]. These problems were studied in various directions: qualitative properties of solutions, spectral problems, various statements of boundary value problems, and numerical investigations.

For example, in [5] the nonlocal boundary value problem

$$
\begin{gathered}
\frac{d^{2} u(t)}{d t^{2}}+A u(t)=f(t), \quad 0 \leq t \leq 1, \\
u(0)=\sum_{r=1}^{n} \alpha_{r} u\left(\lambda_{r}\right)+\varphi, \quad u_{t}(0)=\sum_{r=1}^{n} \beta_{r} u_{t}\left(\lambda_{r}\right)+\psi, \\
0<\lambda_{1} \leq \lambda_{2} \leq \cdots \leq \lambda_{n} \leq 1
\end{gathered}
$$

was investigated. The stability estimates for the solution of the problem were established. The first order of accuracy difference schemes for the approximate solution of this problem was presented. The stability estimates for the solution of these difference schemes were established. Theoretical statements were supported by numerical examples.
The well-posedness of the Cauchy problem, Goursat problem, and boundary value problem for multidimensional hyperbolic equations have been studied extensively in a large cycle of papers (see, e.g., [14-21] and the references therein).

Actually, in paper [14], the Goursat problem for a linear multidimensional hyperbolic equation was investigated. Uniqueness of the solution and weak solvability of the Goursat problem were established.

In paper [15], the existence or nonexistence of global solutions of a multidimensional version of the first Darboux problem for wave equations with power nonlinearity in the conic domain was investigated.

In $[16,17]$, the solvability of an initial-boundary value problem for second order linear hyperbolic equations with a condition on the lateral boundary connecting the values of the solution or the conormal derivative of the solution with the values of some integral operator of the solution was studied. The existence and uniqueness theorems for regular solutions were proved.

In [18-20], the difference schemes for multidimensional hyperbolic equations were investigated. These methods were stable under the inequalities and contain the connection between the grid step sizes of time and space variables.

In [21], the authors develop a finite difference method (FDM) for a multidimensional coupled system of nonlinear parabolic and hyperbolic equations and prove the existence, 
stability, and uniqueness of its solution by a set of theorems. Finally, the proposed method was illustrated by a number of numerical experiments.

The study of difference schemes for hyperbolic equations with nonlocal conditions without using any necessary condition concerning the grid step sizes is of great interest. Such a difference scheme for solving the initial-value problem for abstract hyperbolic equations was studied for the first time in [22]. In the present paper, the following multidimensional hyperbolic equation

$$
\begin{gathered}
\frac{\partial^{2} u(t, x)}{\partial t^{2}}-\sum_{r=1}^{m}\left(a_{r}(x) u_{x_{r}}\right)_{x_{r}}+\sigma u(t, x)=f(t, x), \\
x=\left(x_{1}, \ldots, x_{m}\right) \in \Omega, \quad 0<t<1,
\end{gathered}
$$

with nonlocal integral

$$
\begin{gathered}
u(0, x)=\int_{0}^{1} \alpha(\rho) u(\rho, x) d \rho+\varphi(x), \quad x \in \bar{\Omega}, \\
u_{t}(0, x)=\int_{0}^{1} \beta(\rho) u_{t}(\rho, x) d \rho+\psi(x), \quad x \in \bar{\Omega},
\end{gathered}
$$

and Neumann

$$
\left.\frac{\partial u(t, x)}{\partial \vec{n}}\right|_{S}=0
$$

or nonclassical conditions

$$
\begin{gathered}
\left.u(t, x)\right|_{S_{1}}=0, \\
\left.\frac{\partial u(t, x)}{\partial \vec{n}}\right|_{S_{2}}=0, \quad 0 \leq t \leq 1,
\end{gathered}
$$

under the assumption

$$
\left|1+\int_{0}^{1} \alpha(s) \beta(s) d s\right|>\int_{0}^{1}(|\alpha(s)|+|\beta(s)|) d s
$$

is considered. Here, $\Omega$ is the unit open cube in the $m$ dimensional Euclidean space $\mathbb{R}^{m}$ with boundary $S=S_{1} \cup S_{2}$, $\bar{\Omega}=\Omega \cup S, a_{r}(x)(x \in \Omega), \varphi(x), \psi(x)(x \in \bar{\Omega})$, and $f(t, x)(t \in$ $(0,1), x \in \Omega)$ are given smooth functions, and $a_{r}(x) \geq a>0$. $\vec{n}$ is the normal vector to $\Omega$.

The first and second order of accuracy difference schemes for multidimensional hyperbolic problem (2) are presented. The schemes are shown to be absolutely stable. It is naturally seen that the second order difference schemes are much more advantageous than the first order ones.

\section{Stability of First Order of Accuracy Difference Scheme}

For approximately solving problem (2), first order of accuracy difference scheme

$$
\begin{gathered}
\tau^{-2}\left(u_{k+1}-2 u_{k}+u_{k-1}\right)+A u_{k+1}=f_{k}, \quad f_{k}=f\left(t_{k+1}\right), \\
t_{k+1}=(k+1) \tau, \quad 1 \leq k \leq N-1, \quad N \tau=1, \\
u_{0}=\sum_{j=1}^{N} \alpha\left(t_{j}\right) u_{j} \tau+\varphi, \\
\left(I+\tau^{2} A\right) \tau^{-1}\left(u_{1}-u_{0}\right)=\sum_{j=1}^{N} \beta\left(t_{j}\right)\left(u_{j}-u_{j-1}\right)+\psi
\end{gathered}
$$

is considered. A study of discretization of the nonlocal boundary value problem also permits one to include general difference schemes in applications, if differential operator in space variables $A$ is replaced by difference operator $A_{h}$ that acts in a Hilbert space and is uniformly self-adjoint positive definite in $h$ for $0<h \leq h_{0}$.

The stability estimates of solution of difference scheme (7) are established under the assumption

$$
1>\sum_{j=1}^{N}\left|\alpha\left(t_{j}\right)\right| \tau \sum_{j=1}^{N}\left|\beta\left(t_{j}\right)\right| \tau+\sum_{j=1}^{N}\left|\alpha\left(t_{j}\right)\right| \tau+\sum_{j=1}^{N}\left|\beta\left(t_{j}\right)\right| \tau .
$$

Lemma 1. The following estimates hold [23]:

$$
\begin{array}{rlrl}
\|R\|_{H \mapsto H} & \leq 1, & & \|\widetilde{R}\|_{H \mapsto H} \leq 1, \\
\left\|R \widetilde{R}^{-1}\right\|_{H \mapsto H} \leq 1, & & \left\|\widetilde{R} R^{-1}\right\|_{H \mapsto H} \leq 1, \\
\left\|\tau A^{1 / 2} R\right\|_{H \mapsto H} \leq 1, & & \left\|\tau A^{1 / 2} \widetilde{R}\right\|_{H \mapsto H} \leq 1,
\end{array}
$$

where

$$
R=\left(I+i \tau A^{1 / 2}\right)^{-1}, \quad \widetilde{R}=\left(I-i \tau A^{1 / 2}\right)^{-1} .
$$

Lemma 2. The operator

$$
\begin{aligned}
T= & I+\sum_{j=1}^{N} \beta\left(t_{j}\right) \frac{\tau}{2} R^{2} \widetilde{R}^{2}\left(R^{j}+\widetilde{R}^{j}\right) \\
& -\sum_{j=1}^{N} \alpha\left(t_{j}\right) \frac{\tau}{2}\left[R^{j-1}-\widetilde{R}^{j-1}\right] \\
& -\sum_{j=1}^{N} \alpha\left(t_{j}\right) \tau \sum_{j=1}^{N} \beta\left(t_{j}\right) \tau(R \widetilde{R})^{j+2}
\end{aligned}
$$


has an inverse

$$
\begin{aligned}
T^{-1}= & \left\{I+\sum_{j=1}^{N} \beta\left(t_{j}\right) \frac{\tau}{2} R^{2} \widetilde{R}^{2}\left(R^{j}+\widetilde{R}^{j}\right)\right. \\
& -\sum_{j=1}^{N} \alpha\left(t_{j}\right) \frac{\tau}{2}\left[R^{j-1}-\widetilde{R}^{j-1}\right] \\
& \left.-\sum_{j=1}^{N} \alpha\left(t_{j}\right) \tau \sum_{j=1}^{N} \beta\left(t_{j}\right) \tau(R \widetilde{R})^{j+2}\right\}^{-1}
\end{aligned}
$$

and the following estimate is satisfied:

$$
\begin{aligned}
\left\|T^{-1}\right\|_{H \rightarrow H} \leq(1) \times(1 & -\sum_{j=1}^{N}\left|\alpha\left(t_{j}\right)\right| \tau \sum_{j=1}^{N}\left|\beta\left(t_{j}\right)\right| \tau \\
& \left.-\sum_{j=1}^{N}\left|\beta\left(t_{j}\right)\right| \tau-\sum_{j=1}^{N}\left|\alpha\left(t_{j}\right)\right| \tau\right)^{-1} .
\end{aligned}
$$

Proof. Using formula (11) and the triangle inequality, we can write

$$
\begin{aligned}
\|T\|_{H \rightarrow H} & \\
\geq & -\sum_{j=1}^{N}\left|\alpha\left(t_{j}\right)\right| \tau \sum_{j=1}^{N}\left|\beta\left(t_{j}\right)\right| \tau\left\|(R \widetilde{R})^{j+2}\right\|_{H \rightarrow H} \\
& -\sum_{j=1}^{N}\left|\beta\left(t_{j}\right)\right| \tau \frac{1}{2}\left\|R^{2} \widetilde{R}^{2}\right\|_{H \rightarrow H}\left(\left\|R^{j}\right\|_{H \rightarrow H}+\left\|\widetilde{R}^{j}\right\|_{H \rightarrow H}\right) \\
& -\sum_{j=1}^{N}\left|\alpha\left(t_{j}\right)\right| \tau \frac{1}{2}\left[\left\|R^{j-1}\right\|_{H \rightarrow H}+\left\|\widetilde{R}^{j-1}\right\|_{H \rightarrow H}\right] .
\end{aligned}
$$

Applying the triangle inequality and estimates (9), we get

$$
\begin{aligned}
\|T\|_{H \rightarrow H} \geq & 1-\sum_{j=1}^{N}\left|\alpha\left(t_{j}\right)\right| \tau \sum_{j=1}^{N}\left|\beta\left(t_{j}\right)\right| \tau \\
& -\sum_{j=1}^{N}\left|\beta\left(t_{j}\right)\right| \tau-\sum_{j=1}^{N}\left|\alpha\left(t_{j}\right)\right| \tau .
\end{aligned}
$$

Thus, estimate (13) follows from this estimate. Lemma 2 is proved. The following theorem on the stability estimates for the solution of difference scheme (7) is established.
Theorem 3. Let $\varphi \in D(A), \psi \in D\left(A^{1 / 2}\right)$, and (8) hold. Then, for the solution of difference scheme (7) the following stability estimates hold:

$$
\begin{gathered}
\max _{0 \leq k \leq N}\left\|u_{k}\right\|_{H} \leq M\left\{\sum_{s=1}^{N-1}\left\|A^{-1 / 2} f_{s}\right\|_{H} \tau+\left\|A^{-1 / 2} \psi\right\|_{H}+\|\varphi\|_{H}\right\}, \\
\max _{1 \leq k \leq N}\left\|\tau^{-1}\left(u_{k}-u_{k-1}\right)\right\|_{H}+\max _{0 \leq k \leq N}\left\|A^{1 / 2} u_{k}\right\|_{H} \\
\leq M\left\{\sum_{s=1}^{N-1}\left\|f_{s}\right\|_{H} \tau+\|\psi\|_{H}+\left\|A^{1 / 2} \varphi\right\|_{H}\right\}, \\
\max _{1 \leq k \leq N-1}\left\|\tau^{-2}\left(u_{k+1}-2 u_{k}+u_{k-1}\right)\right\|_{H}+\max _{0 \leq k \leq N}\left\|A u_{k}\right\|_{H} \\
\leq M\left\{\sum_{s=2}^{N-1}\left\|f_{s}-f_{s-1}\right\|_{H}+\left\|f_{1}\right\|_{H}+\left\|A^{1 / 2} \psi\right\|_{H}+\|A \varphi\|_{H}\right\},
\end{gathered}
$$

where $M$ is independent of $f_{s}, 1 \leq s \leq N-1$, and $\varphi, \psi$.

Proof. First, we obtain formula for the solution of difference scheme (7). For the solution of difference scheme

$$
\begin{gathered}
\tau^{-2}\left(u_{k+1}-2 u_{k}+u_{k-1}\right)+A u_{k+1}=f_{k}, \\
f_{k}=f\left(t_{k+1}\right), \quad t_{k+1}=(k+1) \tau, \\
1 \leq k \leq N-1, \quad N \tau=1, \\
u_{0}=\mu, \quad\left(I+\tau^{2} A\right) \tau^{-1}\left(u_{1}-u_{0}\right)=\omega,
\end{gathered}
$$

the following formulas

$$
\begin{gathered}
u_{0}=\mu, \quad u_{1}=\mu+\tau R \widetilde{R} \omega, \\
u_{k}=\frac{1}{2}\left[R^{k-1}+\widetilde{R}^{k-1}\right] \mu+(R-\widetilde{R})^{-1} \tau\left(R^{k}-\widetilde{R}^{k}\right) \omega \\
-\sum_{s=1}^{k-1} \frac{\tau}{2 i} A^{-1 / 2}\left[R^{k-s}-\widetilde{R}^{k-s}\right] f_{s}, \quad 2 \leq k \leq N,
\end{gathered}
$$

were obtained in [22]. Applying formula (20) and nonlocal boundary conditions in (7), we can write formula for $\mu$ and $\omega$

$$
\begin{aligned}
\mu=T^{-1}\{[ & {\left[\alpha(\tau)+\sum_{j=2}^{N} \alpha\left(t_{j}\right)\right] \tau } \\
& \left.\times \sum_{s=1}^{j-1} \frac{\tau}{2 i} A^{-1 / 2}\left[\widetilde{R}^{j-s}-R^{j-s}\right] f_{s}+\varphi\right] \\
& \times\left[I+\sum_{j=1}^{N} \beta\left(t_{j}\right) \frac{\tau}{2} R \widetilde{R}\left(R^{j}+\widetilde{R}^{j}\right)\right]
\end{aligned}
$$




$$
\begin{aligned}
& +\sum_{j=1}^{N} \alpha\left(t_{j}\right) \tau^{2}(R-\widetilde{R})^{-1}\left(R^{j}-\widetilde{R}^{j}\right) \\
& \times R \widetilde{R}\left[\beta(\tau)+\beta(2 \tau)+\sum_{j=3}^{N} \beta\left(t_{j}\right)\right] \frac{\tau^{2}}{2} \\
& \times \sum_{s=1}^{j-2}\left[R^{j-s}-\widetilde{R}^{j-s}\right] f_{s} \\
& \left.-\sum_{j=1}^{N} \beta\left(t_{j}\right) \frac{\tau}{2 i}(R-\widetilde{R}) A^{-1 / 2} f_{j-1}+\psi\right\}, \\
\omega=T & {\left[\begin{array}{l}
\left.I-\sum_{j=1}^{N} \alpha\left(t_{j}\right) \frac{\tau}{2}\left[R^{j-1}-\widetilde{R}^{j-1}\right]\right] \\
\times \\
\times\left[\sum_{j=1} \beta(\tau)+\beta(2 \tau)+\sum_{j=3}^{N} \beta\left(t_{j}\right)\right] \\
\left.+\sum_{j}^{N} \beta(\tau)+\sum_{j=2}^{N} \alpha\left(t_{j}\right)\right] \tau \\
\quad \times \frac{\tau^{2}}{2} \sum_{s=1}^{j-2}\left[R^{j-s}-\widetilde{R}^{j-s}\right] f_{s} \\
\quad-\sum_{j=1}^{N} \beta\left(t_{j}\right) \frac{\tau}{2 i}(R-\widetilde{R}) A^{-1 / 2} f_{j-1}+\psi
\end{array}\right] }
\end{aligned}
$$

Hence, for the solution of nonlocal boundary value problem (7) we have formulas (20), (21), and (22).

Second, let us investigate stability of difference scheme (7). In [22], for the solution of (19) stability estimates

$$
\max _{0 \leq k \leq N}\left\|u_{k}\right\|_{H} \leq M\left\{\sum_{s=1}^{N-1}\left\|A^{-1 / 2} f_{s}\right\|_{H} \tau+\left\|A^{-1 / 2} \omega\right\|_{H}+\|\mu\|_{H}\right\},
$$

$$
\begin{array}{r}
\max _{1 \leq k \leq N}\left\|\tau^{-1}\left(u_{k}-u_{k-1}\right)\right\|_{H}+\max _{0 \leq k \leq N}\left\|A^{1 / 2} u_{k}\right\|_{H} \\
\leq M\left\{\sum_{s=1}^{N-1}\left\|f_{s}\right\|_{H} \tau+\|\omega\|_{H}+\left\|A^{1 / 2} \mu\right\|_{H}\right\},
\end{array}
$$

$$
\begin{aligned}
& \max _{1 \leq k \leq N-1}\left\|\tau^{-2}\left(u_{k+1}-2 u_{k}+u_{k-1}\right)\right\|_{H}+\max _{0 \leq k \leq N}\left\|A u_{k}\right\|_{H} \\
& \leq M\left\{\sum_{s=2}^{N-1}\left\|f_{s}-f_{s-1}\right\|_{H}+\left\|f_{1}\right\|_{H}+\left\|A^{1 / 2} \omega\right\|_{H}+\|A \mu\|_{H}\right\}
\end{aligned}
$$

were established.

First of all, let us find estimate for $\|\mu\|_{H}$. By using formula (21) and estimates (9), we obtain

$$
\|\mu\|_{H} \leq M\left\{\|\varphi\|_{H}+\sum_{j=1}^{N-1}\left\|A^{-1 / 2} f_{j}\right\|_{H} \tau+\left\|A^{-1 / 2} \psi\right\|_{H}\right\} .
$$

And, applying $A^{-1 / 2}$ to formula (22), we get

$$
\begin{aligned}
A^{-1 / 2} \omega=T^{-1}\{[I & \left.-\sum_{j=1}^{N} \alpha\left(t_{j}\right) \frac{\tau}{2}\left[R^{j-1}-\widetilde{R}^{j-1}\right]\right] \\
\times & {\left[\left[\beta(\tau)+\beta(2 \tau)+\sum_{j=3}^{N} \beta\left(t_{j}\right)\right]\right.} \\
& \times \frac{\tau^{2}}{2} \sum_{s=1}^{j-2}\left[R^{j-s}-\widetilde{R}^{j-s}\right] A^{-1 / 2} f_{s} \\
& -\sum_{j=1}^{N} \beta\left(t_{j}\right) \frac{\tau}{2 i}(R-\widetilde{R}) A^{-1} f_{j-1} \\
+ & \sum_{j=1}^{N} \beta\left(t_{j}\right) \frac{i \tau}{2}\left[R^{j-1}-\widetilde{R}^{j-1}\right] \\
\times & {\left[\left[\alpha(\tau)+\sum_{j=2}^{N} \alpha\left(t_{j}\right)\right] \tau\right.} \\
& \left.\left.\times \sum_{s=1}^{-1 / 2} \psi \frac{\tau}{2 i}\left[R^{j-s}-\widetilde{R}^{j-s}\right] A^{-1 / 2} f_{s}-\varphi\right]\right\} .
\end{aligned}
$$

Using the triangle inequality, formula (27), and estimates (9), it follows that

$$
\left\|A^{-1 / 2} \omega\right\|_{H} \leq M\left\{\sum_{j=1}^{N-1}\left\|A^{-1 / 2} f_{j}\right\|_{H} \tau+\left\|A^{-1 / 2} \psi\right\|_{H}+\|\varphi\|_{H}\right\} .
$$

So, estimate (16) follows from estimates (23), (26), and (28). Second, applying $A^{1 / 2}$ to formula (21) and using estimates (9), we get estimate

$$
\left\|A^{1 / 2} \mu\right\|_{H} \leq M\left\{\sum_{j=1}^{N-1}\left\|f_{j}\right\|_{H} \tau+\|\psi\|_{H}+\left\|A^{1 / 2} \varphi\right\|_{H}\right\} .
$$


By using formula (22) and estimates (9), we obtain

$$
\|\omega\|_{H} \leq M\left\{\sum_{j=1}^{N-1}\left\|f_{j}\right\|_{H} \tau+\|\psi\|_{H}+\left\|A^{1 / 2} \varphi\right\|_{H}\right\} .
$$

Using estimates (24), (29), and (30), we obtain estimate (17) for the solution of (7). Third, applying $A$ to formula (21) and using Abel's formula, we can write formula for $A \mu$

$$
\begin{aligned}
A \mu=T^{-1}\{[ & {\left[\alpha(\tau)+\sum_{j=2}^{N} \alpha\left(t_{j}\right)\right] \tau } \\
& \times \sum_{s=2}^{j-1} \frac{1}{2}\left[\widetilde{R}^{j-s}-R^{j-s}\right]\left(f_{s}-f_{s-1}\right) \\
+ & \left.\left(\widetilde{R}^{j-1}-R^{j-1}\right) f_{1}-(\widetilde{R}-R) f_{j-1}+A \varphi\right] \\
\times & {\left[I+\sum_{j=1}^{N} \beta\left(t_{j}\right) \frac{\tau}{2} R \widetilde{R}^{j}\left(R^{j}+\widetilde{R}^{j}\right)\right] } \\
+ & \sum_{j=1}^{N} \alpha\left(t_{j}\right) \tau\left(R^{j}-\widetilde{R}^{j}\right) \\
\times & {\left[\frac{\tau^{2}}{2}(R \widetilde{R})^{2}\left[\beta(\tau)+\beta(2 \tau)+\sum_{j=3}^{N} \beta\left(t_{j}\right)\right]\right.} \\
& +\left(\widetilde{R}^{j-2}-R^{j-2}\right) f_{1}-(\widetilde{R}-R) f_{j-2} \beta \\
& \times \sum_{s=2}^{j-2}\left[\widetilde{R}^{j-s}-R^{j-s}\right]\left(f_{s}-f_{s-1}\right) \\
& \left.\left.\left.+t_{j}\right) f_{j-1}+A^{1 / 2} \psi\right]\right\}
\end{aligned}
$$

It follows from formula (31) and estimates (9) that

$\|A \mu\|_{H}$

$$
\leq M\left\{\sum_{s=2}^{N-1}\left\|f_{s}-f_{s-1}\right\|_{H}+\left\|f_{1}\right\|_{H}+\left\|A^{1 / 2} \psi\right\|_{H}+\|A \varphi\|_{H}\right\} .
$$

Applying $A^{1 / 2}$ to formula (22) and using Abel's formula, we get

$$
\begin{aligned}
& A^{1 / 2} \omega \\
& =T^{-1}\left\{\left[I-\sum_{j=1}^{N} \alpha\left(t_{j}\right) \frac{\tau}{2}\left[R^{j-1}-\widetilde{R}^{j-1}\right]\right]\right.
\end{aligned}
$$

$$
\begin{aligned}
& \times\left[\left[\beta(\tau)+\beta(2 \tau)+\sum_{j=3}^{N} \beta\left(t_{j}\right)\right]\right. \\
& \times \frac{\tau^{2}}{2} \sum_{s=2}^{j-2}\left[\widetilde{R}^{j-s}-R^{j-s}\right] \\
& \times\left(f_{s}-f_{s-1}\right)+\left(\widetilde{R}^{j-2}-R^{j-2}\right) f_{1}-(\widetilde{R}-R) f_{j-1} \\
& \left.+\sum_{j=1}^{N} \beta\left(t_{j}\right) \frac{\tau}{2 i}(R-\widetilde{R}) f_{j-1}+A^{1 / 2} \psi\right] \\
& +\sum_{j=1}^{N} \beta\left(t_{j}\right) \frac{i \tau}{2}\left[R^{j-1}-\widetilde{R}^{j-1}\right] \\
& \times\left[\left[\alpha(\tau)+\sum_{j=2}^{N} \alpha\left(t_{j}\right)\right] \tau\right. \\
& \times \sum_{s=2}^{j-1} \frac{1}{2}\left[\widetilde{R}^{j-s}-R^{j-s}\right]\left(f_{s}-f_{s-1}\right) \\
& \left.\left.+\left(\widetilde{R}^{j-1}-R^{j-1}\right) f_{1}-(\widetilde{R}-R) f_{j-1}\right]-A \varphi\right\},
\end{aligned}
$$

and using the triangle inequality and estimates (9), we obtain the estimate

$$
\begin{aligned}
& \left\|A^{1 / 2} \omega\right\|_{H} \\
& \quad \leq M\left\{\sum_{s=2}^{N-1}\left\|f_{s}-f_{s-1}\right\|_{H}+\left\|f_{1}\right\|_{H}+\left\|A^{1 / 2} \psi\right\|_{H}+\|A \varphi\|_{H}\right\} .
\end{aligned}
$$

Thus, estimate (18) follows from estimates (23), (32), and (34). This is the end of the proof of Theorem 3.

\section{Stability of Second Order of Accuracy Difference Scheme}

Now, we consider the second order accuracy difference scheme for approximate solution of boundary value problem (2)

$$
\begin{gathered}
\tau^{-2}\left(u_{k+1}-2 u_{k}+u_{k-1}\right)+\frac{1}{2} A u_{k}+\frac{1}{4} A\left(u_{k+1}+u_{k-1}\right)=f_{k}, \\
f_{k}=f\left(t_{k}\right), \quad t_{k}=k \tau, \quad 1 \leq k \leq N-1, \quad N \tau=1, \\
u_{0}=\sum_{j=1}^{N} \alpha\left(t_{j}-\frac{\tau}{2}\right)\left[\frac{u_{j}+u_{j-1}}{2}\right] \tau+\varphi,
\end{gathered}
$$




$$
\begin{gathered}
\left(I+\frac{\tau^{2} A}{4}\right) \tau^{-1}\left(u_{1}-u_{0}\right)-\frac{\tau}{2}\left(f_{0}-A u_{0}\right) \\
=\sum_{j=1}^{N} \beta\left(t_{j}-\frac{\tau}{2}\right)\left[u_{j}-u_{j-1}\right]+\psi
\end{gathered}
$$

The stability of solutions of this difference scheme is investigated under the assumption

$$
\begin{aligned}
1> & \sum_{j=1}^{N}\left|\alpha\left(t_{j}-\frac{\tau}{2}\right)\right| \tau+\sum_{j=1}^{N}\left|\beta\left(t_{j}-\frac{\tau}{2}\right)\right| \tau \\
& +\sum_{j=1}^{N}\left|\alpha\left(t_{j}-\frac{\tau}{2}\right)\right| \tau \sum_{j=1}^{N}\left|\beta\left(t_{j}-\frac{\tau}{2}\right)\right| \tau .
\end{aligned}
$$

Lemma 4. The following estimates hold [23]:

$$
\begin{gathered}
\left\|\left(I \pm \frac{i \tau A^{1 / 2}}{2}\right)^{-1}\right\|_{H \mapsto H} \leq 1, \\
\|R\|_{H \mapsto H} \leq 1, \quad\|\widetilde{R}\|_{H \mapsto H} \leq 1, \\
\left\|\left(I \pm i \tau A^{1 / 2}\right)^{-1}\right\|_{H \mapsto H} \leq 1, \\
\left\|\tau A^{1 / 2}\left(I \pm i \tau A^{1 / 2}\right)^{-1}\right\|_{H \mapsto H} \leq 1,
\end{gathered}
$$

where

$$
\begin{aligned}
& R=\left(I-\frac{i \tau A^{1 / 2}}{2}\right)\left(I+\frac{i \tau A^{1 / 2}}{2}\right)^{-1}, \\
& R=\left(I+\frac{i \tau A^{1 / 2}}{2}\right)\left(I-\frac{i \tau A^{1 / 2}}{2}\right)^{-1} .
\end{aligned}
$$

Lemma 5. Suppose that assumption (36) holds. Then, the operator

$$
\begin{aligned}
T=I- & \alpha\left(\frac{\tau}{2}\right)+\left[\alpha\left(\frac{\tau}{2}\right)+\alpha\left(\frac{3 \tau}{2}\right)\right]\left(\frac{R+\widetilde{R}}{2}\right) \\
& +\alpha\left(\frac{3 \tau}{2}\right)\left(\frac{R^{2}+\widetilde{R}^{2}}{2}\right) \\
& \left.+\sum_{j=3}^{N} \alpha\left(t_{j}-\frac{\tau}{2}\right) \frac{\widetilde{R}^{j}+R^{j}+R^{j-1}+\widetilde{R}^{j-1}}{2}\right\} \frac{\tau}{2} \\
- & \left\{\beta\left(\frac{\tau}{2}\right)-\beta\left(\frac{3 \tau}{2}\right)\right] \times\left(I+\frac{\tau^{2} A}{4}\right)^{-1} \tau \\
& +\beta\left(\frac{3 \tau}{2}\right) i A^{-1 / 2} \frac{R^{2}-\widetilde{R}^{2}}{2} \\
& \left.+i A^{-1 / 2} \sum_{j=3}^{N} \beta\left(t_{j}-\frac{\tau}{2}\right) \frac{R^{j}-\widetilde{R}^{j}-R^{j-1}+\widetilde{R}^{j-1}}{2}\right\}
\end{aligned}
$$

$$
\begin{aligned}
+\left\{\left[\beta\left(\frac{\tau}{2}\right)-\beta\left(\frac{3 \tau}{2}\right)\right]\left(I+\frac{\tau^{2} A}{4}\right)^{-1} \tau\right. \\
+\beta\left(\frac{3 \tau}{2}\right) i A^{-1 / 2} \frac{R^{2}-\widetilde{R}^{2}}{2}+i A^{-1 / 2} \\
\left.\quad \times \sum_{j=3}^{N} \beta\left(t_{j}-\frac{\tau}{2}\right) \frac{R^{j}-\widetilde{R}^{j}-R^{j-1}+\widetilde{R}^{j-1}}{2}\right\}
\end{aligned}
$$$$
\times\left\{\alpha\left(\frac{\tau}{2}\right)+\left[\alpha\left(\frac{\tau}{2}\right)+\alpha\left(\frac{3 \tau}{2}\right)\right]\right.
$$$$
\times\left(\frac{R+\widetilde{R}}{2}\right)+\alpha\left(\frac{3 \tau}{2}\right)\left(\frac{R^{2}+\widetilde{R}^{2}}{2}\right)
$$$$
\left.+\sum_{j=3}^{N} \alpha\left(t_{j}-\frac{\tau}{2}\right) \times \frac{\widetilde{R}^{j}+R^{j}+R^{j-1}+\widetilde{R}^{j-1}}{2}\right\} \frac{\tau}{2}
$$$$
-\left\{\left[\beta\left(\frac{\tau}{2}\right)-\beta\left(\frac{3 \tau}{2}\right)\right]\left(\frac{R+\widetilde{R}}{2}\right)\right.
$$$$
+\beta\left(\frac{\tau}{2}\right)+\beta\left(\frac{3 \tau}{2}\right) \frac{R^{2}+\widetilde{R}^{2}}{2}
$$$$
\left.+\sum_{j=3}^{N} \beta\left(t_{j}-\frac{\tau}{2}\right) \frac{R^{j}+\widetilde{R}^{j}-\widetilde{R}^{j-1}-R^{j-1}}{2}\right\}
$$$$
\times\left\{\left[\alpha\left(\frac{\tau}{2}\right)+\alpha\left(\frac{3 \tau}{2}\right)\right] \tau+\alpha\left(\frac{3 \tau}{2}\right) A^{-1 / 2} \frac{R^{2}-\widetilde{R}^{2}}{2 i}\right.
$$$$
\left.+A^{-1 / 2} \sum_{j=3}^{N} \alpha\left(t_{j}-\frac{\tau}{2}\right) \frac{R^{j}-\widetilde{R}^{j}+R^{j-1}-\widetilde{R}^{j-1}}{2 i}\right\} \frac{\tau}{2}
$$

has an inverse $T^{-1}$ and the following estimate is satisfied:

$$
\begin{aligned}
\left\|T^{-1}\right\|_{H \rightarrow H} \leq & {\left[1-\sum_{j=1}^{N}\left|\alpha\left(t_{j}-\frac{\tau}{2}\right)\right| \tau \sum_{j=1}^{N}\left|\beta\left(t_{j}-\frac{\tau}{2}\right)\right| \tau\right.} \\
& \left.-\sum_{j=1}^{N}\left|\beta\left(t_{j}-\frac{\tau}{2}\right)\right| \tau-\sum_{j=1}^{N}\left|\alpha\left(t_{j}-\frac{\tau}{2}\right)\right| \tau\right]^{-1} .
\end{aligned}
$$

Proof. Using formula (39), the triangle inequality, and estimates (37), we obtain

$\|T\|_{H \rightarrow H}$

$\geq 1-\left\{\left|\alpha\left(\frac{\tau}{2}\right)\right|+\left[\left|\alpha\left(\frac{\tau}{2}\right)\right|+\left|\alpha\left(\frac{3 \tau}{2}\right)\right|\right]\left\|\frac{R+\widetilde{R}}{2}\right\|_{H \rightarrow H}\right.$ 


$$
\begin{aligned}
& +\left|\alpha\left(\frac{3 \tau}{2}\right)\right| \times\left\|\frac{R^{2}+\widetilde{R}^{2}}{2}\right\|_{H \rightarrow H} \\
& \left.+\sum_{j=3}^{N}\left|\alpha\left(t_{j}-\frac{\tau}{2}\right)\right|\left\|\frac{\widetilde{R}^{j}+R^{j}+R^{j-1}+\widetilde{R}^{j-1}}{2}\right\|_{H \rightarrow H}\right\} \frac{\tau}{2} \\
& -\left\{\left[\left|\beta\left(\frac{\tau}{2}\right)\right|-\left|\beta\left(\frac{3 \tau}{2}\right)\right|\right]\left\|\left(I+\frac{\tau^{2} A}{4}\right)^{-1}\right\|_{H \rightarrow H} \tau\right. \\
& +\left|\beta\left(\frac{3 \tau}{2}\right)\right| \times\left\|A^{-1 / 2} \frac{R^{2}-\widetilde{R}^{2}}{2}\right\|_{H \rightarrow H} \\
& +\sum_{j=3}^{N}\left|\beta\left(t_{j}-\frac{\tau}{2}\right)\right| \\
& \left.\times\left\|A^{-1 / 2} \frac{R^{j}-\widetilde{R}^{j}-R^{j-1}+\widetilde{R}^{j-1}}{2}\right\|_{H \rightarrow H}\right\} \\
& +\left\{\left[\left|\beta\left(\frac{\tau}{2}\right)\right|-\left|\beta\left(\frac{3 \tau}{2}\right)\right|\right]\left\|\left(I+\frac{\tau^{2} A}{4}\right)^{-1}\right\|_{H \rightarrow H} \tau\right. \\
& +\left|\beta\left(\frac{3 \tau}{2}\right)\right| \times\left\|A^{-1 / 2} \frac{R^{2}-\widetilde{R}^{2}}{2}\right\|_{H \rightarrow H} \\
& +\sum_{j=3}^{N}\left|\beta\left(t_{j}-\frac{\tau}{2}\right)\right| \\
& \left.\times\left\|A^{-1 / 2} \frac{R^{j}-\widetilde{R}^{j}-R^{j-1}+\widetilde{R}^{j-1}}{2}\right\|_{H \rightarrow H}\right\} \\
& \times\left\{\left|\alpha\left(\frac{\tau}{2}\right)\right|+\left[\left|\alpha\left(\frac{\tau}{2}\right)\right|+\left|\alpha\left(\frac{3 \tau}{2}\right)\right|\right]|| \frac{R+\widetilde{R}}{2} \|_{H \rightarrow H}\right. \\
& +\left|\alpha\left(\frac{3 \tau}{2}\right)\right| \times\left\|\frac{R^{2}+\widetilde{R}^{2}}{2}\right\|_{H \rightarrow H} \\
& \left.+\sum_{j=3}^{N}\left|\alpha\left(t_{j}-\frac{\tau}{2}\right)\right|\left\|\frac{\tilde{R}^{j}+R^{j}+R^{j-1}+\widetilde{R}^{j-1}}{2}\right\|_{H \rightarrow H}\right\} \frac{\tau}{2} \\
& -\left\{\left[\left|\beta\left(\frac{\tau}{2}\right)\right|-\left|\beta\left(\frac{3 \tau}{2}\right)\right|\right]\left\|\frac{R+\widetilde{R}}{2}\right\|_{H \rightarrow H}\right. \\
& +\left|\beta\left(\frac{\tau}{2}\right)\right|+\left|\beta\left(\frac{3 \tau}{2}\right)\right| \times\left\|\frac{R^{2}+\widetilde{R}^{2}}{2}\right\|_{H \rightarrow H} \\
& \left.+\sum_{j=3}^{N}\left|\beta\left(t_{j}-\frac{\tau}{2}\right)\right|\left\|\frac{R^{j}+\widetilde{R}^{j}-\widetilde{R}^{j-1}-R^{j-1}}{2}\right\|_{H \rightarrow H}\right\} \\
& \times\left\{\left[\left|\alpha\left(\frac{\tau}{2}\right)\right|+\left|\alpha\left(\frac{3 \tau}{2}\right)\right|\right] \tau\right.
\end{aligned}
$$

$$
\begin{aligned}
& +\left|\alpha\left(\frac{3 \tau}{2}\right)\right|\left\|A^{-1 / 2} \frac{R^{2}-\widetilde{R}^{2}}{2}\right\|_{H \rightarrow H} \\
& +\sum_{j=3}^{N}\left|\alpha\left(t_{j}-\frac{\tau}{2}\right)\right| \\
& \left.\times\left\|A^{-1 / 2} \frac{R^{j}-\widetilde{R}^{j}+R^{j-1}-\widetilde{R}^{j-1}}{2}\right\|_{H \rightarrow H}\right\} \frac{\tau}{2} \\
& \geq 1-\left\{\left|\alpha\left(\frac{\tau}{2}\right)\right|+\left|\alpha\left(\frac{3 \tau}{2}\right)\right|+\sum_{j=3}^{N}\left|\alpha\left(t_{j}-\frac{\tau}{2}\right)\right|\right\} \tau \\
& -\left\{\left|\beta\left(\frac{\tau}{2}\right)\right|+\left|\beta\left(\frac{3 \tau}{2}\right)\right|+\sum_{j=3}^{N}\left|\beta\left(t_{j}-\frac{\tau}{2}\right)\right|\right\} \tau \\
& -\left\{\left|\beta\left(\frac{\tau}{2}\right)\right|+\left|\beta\left(\frac{3 \tau}{2}\right)\right|+\sum_{j=3}^{N}\left|\beta\left(t_{j}-\frac{\tau}{2}\right)\right|\right\} \tau \\
& \times\left\{\left|\alpha\left(\frac{\tau}{2}\right)\right|+\left|\alpha\left(\frac{3 \tau}{2}\right)\right|+\sum_{j=3}^{N}\left|\alpha\left(t_{j}-\frac{\tau}{2}\right)\right|\right\} \tau \\
& \geq 1-\sum_{j=1}^{N}\left|\alpha\left(t_{j}-\frac{\tau}{2}\right)\right| \tau-\sum_{j=1}^{N}\left|\beta\left(t_{j}-\frac{\tau}{2}\right)\right| \tau \\
& -\sum_{j=1}^{N}\left|\alpha\left(t_{j}-\frac{\tau}{2}\right)\right| \tau \sum_{j=1}^{N}\left|\beta\left(t_{j}-\frac{\tau}{2}\right)\right| \tau .
\end{aligned}
$$

Estimate (40) follows from this estimate. Lemma 5 is proved.

Theorem 6. Let $\varphi \in D(A), \psi \in D\left(A^{1 / 2}\right)$, and assumption (36) hold. For the solution of difference scheme (3) the following stability estimates

$$
\begin{gathered}
\max _{0 \leq k \leq N}\left\|u_{k}\right\|_{H} \leq M\left\{\sum_{s=0}^{N-1}\left\|A^{-1 / 2} f_{s}\right\|_{H} \tau+\left\|A^{-1 / 2} \psi\right\|_{H}+\|\varphi\|_{H}\right\}, \\
\max _{1 \leq k \leq N}\left\|\tau^{-1}\left(u_{k}-u_{k-1}\right)\right\|_{H}+\max _{0 \leq k \leq N}\left\|A^{1 / 2} u_{k}\right\|_{H} \\
\leq M\left\{\sum_{s=0}^{N-1}\left\|f_{s}\right\|_{H} \tau+\left\|A^{1 / 2} \varphi\right\|_{H}+\|\psi\|_{H}\right\}, \\
\max _{1 \leq k \leq N-1}\left\|\tau^{-2}\left(u_{k+1}-2 u_{k}+u_{k-1}\right)\right\|_{H}+\max _{1 \leq k \leq N}\left\|A \frac{u_{k}+u_{k-1}}{2}\right\|_{H} \\
\leq M\left\{\sum_{s=1}^{N-1}\left\|f_{s}-f_{s-1}\right\|_{H}+\left\|f_{0}\right\|_{H}+\left\|A^{1 / 2} \psi\right\|_{H}+\|A \varphi\|_{H}\right\}
\end{gathered}
$$

are valid, where $M$ is independent of $f_{s}, 0 \leq s \leq N-1$, and $\varphi, \psi$. 
Proof. We obtain formula for the solution of difference scheme (3). For the solution of difference scheme

$$
\begin{array}{r}
\tau^{-2}\left(u_{k+1}-2 u_{k}+u_{k-1}\right)+\frac{1}{2} A u_{k}+\frac{1}{4} A\left(u_{k+1}+u_{k-1}\right)=f_{k}, \\
f_{k}=f\left(t_{k}\right), \quad t_{k}=k \tau, \\
1 \leq k \leq N-1, \quad N \tau=1, \\
u_{0}=\mu, \quad\left(I+\frac{\tau^{2} A}{4}\right) \tau^{-1}\left(u_{1}-u_{0}\right)+\frac{\tau}{2}\left(A u_{0}-f_{0}\right)=\omega,
\end{array}
$$

the following formulas

$$
\begin{aligned}
u_{0}= & \mu, \\
u_{1}= & \left(I+\frac{\tau^{2} A}{4}\right)^{-1}\left[\left(I-\frac{\tau^{2} A}{4}\right) \mu+\tau \omega+\frac{\tau}{2} f_{0}\right] \\
u_{k}= & \frac{1}{2}\left[R^{k}+\widetilde{R}^{k}\right] \mu+\frac{1}{2 i} A^{-1 / 2}\left[R^{k}-\widetilde{R}^{k}\right]\left(\omega+\frac{\tau}{2} f_{0}\right) \\
& -\sum_{s=1}^{k-1} \frac{\tau}{2 i} A^{-1 / 2}\left[R^{k-s}-\widetilde{R}^{k-s}\right] f_{s}, \quad 2 \leq k \leq N
\end{aligned}
$$

were obtained in [22]. Applying formula (46) and nonlocal boundary conditions in (3), we obtain formulas

$$
\begin{aligned}
& \mu=T^{-1}\left\{\left[I-\left[\left[\beta\left(\frac{\tau}{2}\right)-\beta\left(\frac{3 \tau}{2}\right)\right]\left(I+\frac{\tau^{2} A}{4}\right)^{-1} \tau\right.\right.\right. \\
& +\beta\left(\frac{3 \tau}{2}\right) i A^{-1 / 2} \frac{R^{2}-\widetilde{R}^{2}}{2} \\
& +\sum_{j=3}^{N} \beta\left(t_{j}-\frac{\tau}{2}\right) i A^{-1 / 2} \\
& \left.\left.\times \frac{R^{j}-\widetilde{R}^{j}+\widetilde{R}^{j-1}-R^{j-1}}{2}\right]\right] \\
& \times\left\{\left\{\alpha\left(\frac{\tau}{2}\right)+\alpha\left(\frac{3 \tau}{2}\right)+\alpha\left(\frac{3 \tau}{2}\right) A^{-1 / 2} \frac{R^{2}-\widetilde{R}^{2}}{2 i}\right.\right. \\
& +\sum_{j=3}^{N} \alpha\left(t_{j}-\frac{\tau}{2}\right) A^{-1 / 2} \\
& \left.\times \frac{R^{j}-\widetilde{R}^{j}+R^{j-1}-\widetilde{R}^{j-1}}{2 i}\right\} \frac{\tau^{2}}{4} f_{0} \\
& -\alpha\left(\frac{3 \tau}{2}\right) \frac{\tau^{2}}{4 i} A^{-1 / 2}(R-\widetilde{R}) f_{1}-\sum_{j=3}^{N} \alpha\left(t_{j}-\frac{\tau}{2}\right) \frac{\tau}{2}
\end{aligned}
$$

$$
\begin{aligned}
& \times\left[\sum_{s=1}^{j-2} \frac{\tau}{2 i} A^{-1 / 2}\left[R^{j-s}-\widetilde{R}^{j-s}+R^{j-s-1}-\widetilde{R}^{j-s-1}\right] f_{s}\right. \\
& \left.\left.+\frac{\tau}{2 i}(R-\widetilde{R}) f_{j-1}\right]+\varphi\right\} \\
& +\left\{\left[\alpha\left(\frac{\tau}{2}\right)+\alpha\left(\frac{3 \tau}{2}\right)\right] \tau+\alpha\left(\frac{3 \tau}{2}\right) A^{-1 / 2} \frac{R^{2}-\widetilde{R}^{2}}{2 i}\right. \\
& \left.+A^{-1 / 2} \sum_{j=3}^{N} \alpha\left(t_{j}-\frac{\tau}{2}\right) \frac{R^{j}-\widetilde{R}^{j}+R^{j-1}-\widetilde{R}^{j-1}}{2 i}\right\} \frac{\tau}{2} \\
& \times\left\{\left\{\left[\beta\left(\frac{\tau}{2}\right)-\beta\left(\frac{3 \tau}{2}\right)\right] \frac{\tau^{2}}{2}\left(I+\frac{\tau^{2} A}{4}\right)^{-1}\right.\right. \\
& +\frac{\tau}{2} i A^{-1 / 2} \beta\left(\frac{3 \tau}{2}\right) \frac{R^{2}-\widetilde{R}^{2}}{2} \\
& +\sum_{j=3}^{N} \frac{\tau}{2} \beta\left(t_{j}-\frac{\tau}{2}\right) i A^{-1 / 2} \\
& \left.\times \frac{R^{j}-\widetilde{R}^{j}+\widetilde{R}^{j-1}-R^{j-1}}{2}\right\} f_{0} \\
& +i \tau A^{-1 / 2} \frac{R-\widetilde{R}}{2} \\
& \times\left[\beta\left(\frac{3 \tau}{2}\right) f_{1}+\sum_{j=3}^{N} \beta\left(t_{j}-\frac{\tau}{2}\right) f_{j-1}\right] \\
& +\frac{i \tau}{2} A^{-1 / 2} \sum_{j=3}^{N} \beta\left(t_{j}-\frac{\tau}{2}\right) \\
& \left.\left.\times \sum_{s=1}^{j-2}\left[R^{j-s}-\widetilde{R}^{j-s}+\widetilde{R}^{j-s-1}-R^{j-s-1}\right] f_{s}+\psi\right\}\right\} \\
& \omega=T^{-1}\left\{\left\{I-\left[\alpha\left(\frac{\tau}{2}\right)+\left[\alpha\left(\frac{\tau}{2}\right)+\alpha\left(\frac{3 \tau}{2}\right)\right]\left(\frac{R+\widetilde{R}}{2}\right)\right.\right.\right. \\
& +\alpha\left(\frac{3 \tau}{2}\right)\left(\frac{R^{2}+\widetilde{R}^{2}}{2}\right) \\
& \left.\left.+\sum_{j=3}^{N} \alpha\left(t_{j}-\frac{\tau}{2}\right) \frac{\widetilde{R}^{j}+R^{j}+R^{j-1}+\widetilde{R}^{j-1}}{2}\right] \frac{\tau}{2}\right\} \\
& \times\left\{\left[\beta\left(\frac{\tau}{2}\right)-\beta\left(\frac{3 \tau}{2}\right)\right] \frac{\tau^{2}}{2}\left(I+\frac{\tau^{2} A}{4}\right)^{-1}\right. \\
& +\frac{\tau}{2} i A^{-1 / 2} \beta\left(\frac{3 \tau}{2}\right) \times \frac{R^{2}-\widetilde{R}^{2}}{2}
\end{aligned}
$$




$$
\begin{aligned}
& +\sum_{j=3}^{N} \frac{\tau}{2} \beta\left(t_{j}-\frac{\tau}{2}\right) i A^{-1 / 2} \\
& \left.\times \frac{R^{j}-\widetilde{R}^{j}+\widetilde{R}^{j-1}-R^{j-1}}{2}\right\} f_{0} \\
& +\left\{i \tau A^{-1 / 2} \frac{R-\widetilde{R}}{2}\right. \\
& \times\left[\beta\left(\frac{3 \tau}{2}\right) f_{1}+\sum_{j=3}^{N} \beta\left(t_{j}-\frac{\tau}{2}\right) f_{j-1}\right] \\
& +\frac{i \tau}{2} A^{-1 / 2} \sum_{j=3}^{N} \beta\left(t_{j}-\frac{\tau}{2}\right) \\
& \times \sum_{s=1}^{j-2}\left[R^{j-s}-\widetilde{R}^{j-s}+\widetilde{R}^{j-s-1}\right. \\
& \left.\left.-R^{j-s-1}\right] f_{s}+\psi\right\} \\
& +\left\{\left[\beta\left(\frac{\tau}{2}\right)-\beta\left(\frac{3 \tau}{2}\right)\right]\left(I+\frac{\tau^{2} A}{4}\right)^{-1}\right. \\
& \times\left(I-\frac{\tau^{2} A}{4}\right)-\beta\left(\frac{\tau}{2}\right) \\
& +\beta\left(\frac{3 \tau}{2}\right) \frac{R^{2}+\widetilde{R}^{2}}{2} \\
& +\sum_{j=3}^{N} \beta\left(t_{j}-\frac{\tau}{2}\right) \\
& \left.\times \frac{R^{j}-R^{j-1}+\widetilde{R}^{j}-\widetilde{R}^{j-1}}{2}\right\} \\
& \times\left\{\left\{\alpha\left(\frac{\tau}{2}\right)+\alpha\left(\frac{3 \tau}{2}\right)\right.\right. \\
& +\alpha\left(\frac{3 \tau}{2}\right) A^{-1 / 2} \frac{R^{2}-\widetilde{R}^{2}}{2 i} \\
& +\sum_{j=3}^{N} \alpha\left(t_{j}-\frac{\tau}{2}\right) A^{-1 / 2} \\
& \left.\times \frac{R^{j}-\widetilde{R}^{j}+R^{j-1}-\widetilde{R}^{j-1}}{2 i}\right\} \frac{\tau^{2}}{4} f_{0} \\
& -\alpha\left(\frac{3 \tau}{2}\right) \frac{\tau^{2}}{4 i} A^{-1 / 2}(R-\widetilde{R}) f_{1} \\
& -\sum_{j=3}^{N} \alpha\left(t_{j}-\frac{\tau}{2}\right) \frac{\tau}{2}
\end{aligned}
$$

Hence, for the solution of nonlocal boundary value problem (3) we have formulas (46), (47), and (48).

Now, let us investigate the stability of difference scheme (3). In [22], for the solution of (45) the following stability estimates

$$
\begin{gathered}
\max _{0 \leq k \leq N}\left\|u_{k}\right\|_{H} \leq M\left\{\sum_{s=0}^{N-1}\left\|A^{-1 / 2} f_{s}\right\|_{H} \tau+\left\|A^{-1 / 2} \omega\right\|_{H}+\|\mu\|_{H}\right\}, \\
\max _{1 \leq k \leq N}\left\|\tau^{-1}\left(u_{k}-u_{k-1}\right)\right\|_{H}+\max _{0 \leq k \leq N}\left\|A^{1 / 2} u_{k}\right\|_{H} \\
\leq M\left\{\sum_{s=0}^{N-1}\left\|f_{s}\right\|_{H} \tau+\|\omega\|_{H}+\left\|A^{1 / 2} \mu\right\|_{H}\right\}, \\
\max _{1 \leq k \leq N-1}\left\|\tau^{-2}\left(u_{k+1}-2 u_{k}+u_{k-1}\right)\right\|_{H}+\max _{1 \leq k \leq N}\left\|A \frac{u_{k}+u_{k-1}}{2}\right\|_{H} \\
\leq M\left\{\sum_{s=1}^{N-1}\left\|f_{s}-f_{s-1}\right\|_{H}+\left\|f_{0}\right\|_{H}+\left\|A^{1 / 2} \omega\right\|_{H}+\|A \mu\|_{H}\right\}
\end{gathered}
$$

were established. Now, from formula (47) and estimates (37) it follows that

$$
\|\mu\|_{H} \leq M\left\{\sum_{s=0}^{N-1}\left\|A^{-1 / 2} f_{s}\right\|_{H} \tau+\left\|A^{-1 / 2} \psi\right\|_{H}+\|\varphi\|_{H}\right\} .
$$

Applying $A^{-1 / 2}$ to formula (48), we get

$$
\begin{array}{r}
A^{-1 / 2} \omega \\
=T^{-1}\left\{\left\{I-\left[\alpha\left(\frac{\tau}{2}\right)+\left[\alpha\left(\frac{\tau}{2}\right)+\alpha\left(\frac{3 \tau}{2}\right)\right]\left(\frac{R+\widetilde{R}}{2}\right)\right.\right.\right. \\
+\alpha\left(\frac{3 \tau}{2}\right)\left(\frac{R^{2}+\widetilde{R}^{2}}{2}\right) \\
+\sum_{j=3}^{N} \alpha\left(t_{j}-\frac{\tau}{2}\right) \\
\left.\left.\quad \times \frac{\widetilde{R}^{j}+R^{j}+R^{j-1}+\widetilde{R}^{j-1}}{2}\right] \frac{\tau}{2}\right\}
\end{array}
$$




$$
\begin{aligned}
& \times\left\{\left\{\left[\beta\left(\frac{\tau}{2}\right)-\beta\left(\frac{3 \tau}{2}\right)\right] \frac{\tau^{2}}{2}\left(I+\frac{\tau^{2} A}{4}\right)^{-1}\right.\right. \\
& +\frac{\tau}{2} i A^{-1 / 2} \beta\left(\frac{3 \tau}{2}\right) \frac{R^{2}-\widetilde{R}^{2}}{2} \\
& +\sum_{j=3}^{N} \frac{\tau}{2} \beta\left(t_{j}-\frac{\tau}{2}\right) i A^{-1 / 2} \\
& \left.\times \frac{R^{j}-\widetilde{R}^{j}+\widetilde{R}^{j-1}-R^{j-1}}{2}\right\} A^{-1 / 2} f_{0} \\
& +i \tau A^{-1 / 2} \frac{R-\widetilde{R}}{2} \\
& \times\left[\beta\left(\frac{3 \tau}{2}\right) A^{-1 / 2} f_{1}+\sum_{j=3}^{N} \beta\left(t_{j}-\frac{\tau}{2}\right) A^{-1 / 2} f_{j-1}\right] \\
& +\frac{i \tau}{2} \sum_{j=3}^{N} \beta\left(t_{j}-\frac{\tau}{2}\right) \\
& \times \sum_{s=1}^{j-2}\left[R^{j-s}-\widetilde{R}^{j-s}+\widetilde{R}^{j-s-1}-R^{j-s-1}\right] A^{-1 / 2} f_{s} \\
& \left.+A^{-1 / 2} \psi\right\} \\
& +\left\{\left[\beta\left(\frac{\tau}{2}\right)-\beta\left(\frac{3 \tau}{2}\right)\right] A^{-1 / 2} \frac{R+\widetilde{R}}{2}-\beta\left(\frac{\tau}{2}\right)\right. \\
& +\beta\left(\frac{3 \tau}{2}\right) A^{-1 / 2} \frac{R^{2}+\widetilde{R}^{2}}{2} \\
& +\sum_{j=3}^{N} \beta\left(t_{j}-\frac{\tau}{2}\right) A^{-1 / 2} \\
& \left.\times \frac{R^{j}-R^{j-1}+\widetilde{R}^{j}-\widetilde{R}^{j-1}}{2}\right\} \\
& \times\left\{\left\{\alpha\left(\frac{\tau}{2}\right)+\alpha\left(\frac{3 \tau}{2}\right)\right.\right. \\
& +\alpha\left(\frac{3 \tau}{2}\right) A^{-1 / 2} \frac{R^{2}-\widetilde{R}^{2}}{2 i} \\
& +\sum_{j=3}^{N} \alpha\left(t_{j}-\frac{\tau}{2}\right) A^{-1 / 2} \\
& \left.\times \frac{R^{j}-\widetilde{R}^{j}+R^{j-1}-\widetilde{R}^{j-1}}{2 i}\right\} \frac{\tau^{2}}{4} f_{0} \\
& -\alpha\left(\frac{3 \tau}{2}\right) \frac{\tau^{2}}{4 i} A^{-1 / 2}(R-\widetilde{R}) f_{1}
\end{aligned}
$$$$
-\sum_{j=3}^{N} \alpha\left(t_{j}-\frac{\tau}{2}\right) \frac{\tau^{2}}{4 i}(R-\widetilde{R}) f_{j-1}
$$$$
-\sum_{j=3}^{N} \alpha\left(t_{j}-\frac{\tau}{2}\right) \frac{\tau}{2}
$$$$
\times \sum_{s=1}^{j-2} \frac{\tau}{2 i} A^{-1 / 2}\left[R^{j-s}-\widetilde{R}^{j-s}+R^{j-s-1}\right.
$$$$
\left.\left.\left.-\widetilde{R}^{j-s-1}\right] f_{s}+\varphi\right\}\right\}
$$

and using estimates (37), we obtain

$$
\left\|A^{-1 / 2} \omega\right\|_{H} \leq M\left\{\sum_{s=0}^{N-1}\left\|A^{-1 / 2} f_{s}\right\|_{H} \tau+\left\|A^{-1 / 2} \psi\right\|_{H}+\|\varphi\|_{H}\right\} .
$$

So, using estimates (49), (52), and (54), we obtain (42) for the solution of (3). Applying $A^{1 / 2}$ to formula (47), we get

$$
\begin{gathered}
A^{1 / 2} \mu \\
=T^{-1}\left\{\left[I-\left[\left[\beta\left(\frac{\tau}{2}\right)-\beta\left(\frac{3 \tau}{2}\right)\right]\left(I+\frac{\tau^{2} A}{4}\right)^{-1} \tau\right.\right.\right. \\
+\beta\left(\frac{3 \tau}{2}\right) i A^{-1 / 2} \frac{R^{2}-\widetilde{R}^{2}}{2} \\
+\sum_{j=3}^{N} \beta\left(t_{j}-\frac{\tau}{2}\right) i A^{-1 / 2} \\
\times\left\{\left[\alpha\left(\frac{\tau}{2}\right)+\alpha\left(\frac{R^{j}-\widetilde{R}^{j}+\widetilde{R}^{j-1}-R^{j-1}}{2}\right]\right)+\alpha\left(\frac{3 \tau}{2}\right) \frac{R^{2}-\widetilde{R}^{2}}{2 i}\right. \\
+\sum_{j=3}^{N} \alpha\left(t_{j}-\frac{\tau}{2}\right) \\
\left.\times \frac{R^{j}-\widetilde{R}^{j}+R^{j-1}-\widetilde{R}^{j-1}}{2 i}\right] \frac{\tau^{2}}{4} f_{0} \\
-\alpha\left(\frac{3 \tau}{2}\right) \frac{\tau^{2}}{4 i}(R-\widetilde{R}) f_{1} \\
-\sum_{j=3}^{N} \alpha\left(t_{j}-\frac{\tau}{2}\right) \frac{\tau^{2}}{4 i}(R-\widetilde{R}) f_{j-1}+A^{1 / 2} \varphi
\end{gathered}
$$




$$
\begin{aligned}
& \quad-\sum_{j=3}^{N} \alpha\left(t_{j}-\frac{\tau}{2}\right) \frac{\tau}{2} \\
& \times \sum_{s=1}^{j-2} \frac{\tau}{2 i}\left[R^{j-s}-\widetilde{R}^{j-s}+R^{j-s-1}\right. \\
& +\left[\left[\alpha\left(\frac{\tau}{2}\right)+\alpha\left(\frac{3 \tau}{2}\right)\right] \tau+\alpha\left(\frac{3 \tau}{2}\right) \frac{R^{2}-\widetilde{R}^{2}}{2 i}\right. \\
& \left.+\sum_{j=3}^{N} \alpha\left(t_{j}-\frac{\tau}{2}\right) \frac{R^{j}-\widetilde{R}^{j}+R^{j-1}-\widetilde{R}^{j-1}}{2 i}\right] \frac{\tau}{2} \\
& \times\left\{\left[\beta\left(\frac{\tau}{2}\right)-\beta\left(\frac{3 \tau}{2}\right)\right] \frac{\tau^{2}}{2}\left(I+\frac{\tau^{2} A}{4}\right)^{-1}\right. \\
& +\left\{\frac{\tau}{2} i A^{-1 / 2} \beta\left(\frac{3 \tau}{2}\right) \frac{R^{2}-\widetilde{R}^{2}}{2}\right. \\
& \quad+\sum_{s=1}^{N}\left[R^{j-s}-\widetilde{R}^{j-s}+\widetilde{R}^{j-s-1}\right. \\
& +\sum_{j=3}^{N} \frac{\tau}{2} \beta\left(t_{j}-\frac{\tau}{2}\right) i A^{-1 / 2} \\
& \left.\left.+i \tau A^{-1 / 2} \frac{R-\widetilde{R}^{j}}{2}\right) f_{1}+\sum_{j=3}^{N} \beta\left(t_{j}-\frac{\tau}{2}\right) f_{j-1}\right] \\
& \left.\left.\left.\quad \times \frac{R^{j}-\widetilde{R}^{j}+\widetilde{R}^{j-1}-R^{j-1}}{2}\right\} f_{s}+\psi\right\}\right\}
\end{aligned}
$$

From the last formula and estimates (37) it follows that

$$
\left\|A^{1 / 2} \mu\right\|_{H} \leq M\left\{\sum_{s=0}^{N-1}\left\|f_{s}\right\|_{H} \tau+\|\psi\|_{H}+\left\|A^{1 / 2} \varphi\right\|_{H}\right\} .
$$

Using formula (48), the triangle inequality, and estimates (37), we obtain

$$
\|\omega\|_{H} \leq M\left\{\sum_{s=0}^{N-1}\left\|f_{s}\right\|_{H} \tau+\|\psi\|_{H}+\left\|A^{1 / 2} \varphi\right\|_{H}\right\} .
$$

Thus, estimate (43) follows from estimates (50), (56), and (57). Applying $A$ to (47) and using Abel's formula, we obtain

$A \mu$

$$
\begin{aligned}
& =T^{-1}\left\{\left[I-\left[\left[\beta\left(\frac{\tau}{2}\right)-\beta\left(\frac{3 \tau}{2}\right)\right]\left(I+\frac{\tau^{2} A}{4}\right)^{-1} \tau\right.\right.\right. \\
& +\beta\left(\frac{3 \tau}{2}\right) \times i A^{-1 / 2} \frac{R^{2}-\widetilde{R}^{2}}{2} \\
& +\sum_{j=3}^{N} \beta\left(t_{j}-\frac{\tau}{2}\right) i A^{-1 / 2} \\
& \left.\left.\times \frac{R^{j}-\widetilde{R}^{j}+\widetilde{R}^{j-1}-R^{j-1}}{2}\right]\right] \\
& \times\left[\alpha\left(\frac{\tau}{2}\right)+\alpha\left(\frac{3 \tau}{2}\right)+\alpha\left(\frac{3 \tau}{2}\right) A^{1 / 2} \frac{R^{2}-\widetilde{R}^{2}}{2 i}\right. \\
& +\sum_{j=3}^{N} \alpha\left(t_{j}-\frac{\tau}{2}\right) A^{1 / 2} \\
& \left.\times \frac{R^{j}-\widetilde{R}^{j}+R^{j-1}-\widetilde{R}^{j-1}}{2 i}\right] \frac{\tau^{2}}{4} f_{0} \\
& -\alpha\left(\frac{3 \tau}{2}\right) \times \frac{\tau^{2}}{4 i} A^{1 / 2}(R-\widetilde{R}) f_{1} \\
& -\sum_{j=3}^{N} \alpha\left(t_{j}-\frac{\tau}{2}\right) \frac{\tau^{2}}{4 i} A^{1 / 2}(R-\widetilde{R}) f_{j-1}+A \varphi \\
& -\sum_{j=3}^{N} \alpha\left(t_{j}-\frac{\tau}{2}\right) \frac{\tau}{4} \\
& \times\left[\sum _ { s = 1 } ^ { j - 2 } \left[\left(I-\frac{i \tau A^{1 / 2}}{2}\right) R^{j-s}\right.\right. \\
& \left.+\left(I+\frac{i \tau A^{1 / 2}}{2}\right) \widetilde{R}^{j-s}\right] \\
& \times\left(f_{s}-f_{s-1}\right)-\left[\left(I-\frac{i \tau A^{1 / 2}}{2}\right) R^{j-s}\right. \\
& \left.+\left(I+\frac{i \tau A^{1 / 2}}{2}\right) \widetilde{R}^{j-s}\right] f_{0}
\end{aligned}
$$$$
\left.+2 f_{j-2}\right]
$$$$
-\sum_{j=3}^{N} \alpha\left(t_{j}-\frac{\tau}{2}\right) \frac{\tau}{4}
$$ 


$$
\begin{aligned}
& \times\left[\sum _ { s = 1 } ^ { j - 2 } \left[\left(I-\frac{i \tau A^{1 / 2}}{2}\right) R^{j-s-1}\right.\right. \\
& -\left[\left(I-\frac{i \tau A^{1 / 2}}{2}\right) R^{j-s}\right. \\
& \left.+\left(I+\frac{i \tau A^{1 / 2}}{2}\right) \widetilde{R}^{j-s-1}\right]\left(f_{s}-f_{s-1}\right) \\
& -\left[\left(I-\frac{i \tau A^{1 / 2}}{2}\right) R^{j-s-1}\right. \\
& \left.\left.+\left(I+\frac{i \tau A^{1 / 2}}{2}\right) \widetilde{R}^{j-s-1}\right] f_{0}+2 f_{j-2}\right] \\
& +\left[\left[\alpha\left(\frac{\tau}{2}\right)+\alpha\left(\frac{3 \tau}{2}\right)\right] \tau\right. \\
& +\alpha\left(\frac{3 \tau}{2}\right) \frac{R^{2}-\widetilde{R}^{2}}{2 i} \\
& +\sum_{j=3}^{N} \alpha\left(t_{j}-\frac{\tau}{2}\right) \\
& \left.\times \frac{R^{j}-\widetilde{R}^{j}+R^{j-1}-\widetilde{R}^{j-1}}{2 i}\right] \frac{\tau}{2} \\
& \times\left\{\left[\left[\beta\left(\frac{\tau}{2}\right)-\beta\left(\frac{3 \tau}{2}\right)\right] \frac{\tau^{2}}{2} A^{1 / 2}\left(I+\frac{\tau^{2} A}{4}\right)^{-1}\right.\right.
\end{aligned}
$$$$
+\frac{\tau}{2} i \beta\left(\frac{3 \tau}{2}\right) \frac{R^{2}-\widetilde{R}^{2}}{2}
$$$$
+\sum_{j=3}^{N} \frac{\tau}{2} \beta\left(t_{j}-\frac{\tau}{2}\right) i
$$$$
\left.\times \frac{R^{j}-\widetilde{R}^{j}+\widetilde{R}^{j-1}-R^{j-1}}{2}\right] f_{0}
$$$$
+i \tau \frac{R-\widetilde{R}}{2}\left[\beta\left(\frac{3 \tau}{2}\right) f_{1}\right.
$$$$
\left.+\sum_{j=3}^{N} \beta\left(t_{j}-\frac{\tau}{2}\right) f_{j-1}\right]+A^{1 / 2} \psi
$$$$
-\sum_{j=3}^{N} \beta\left(t_{j}-\frac{\tau}{2}\right) \frac{\tau}{4}
$$$$
\times\left[\sum _ { s = 1 } ^ { j - 2 } \left[\left(I-\frac{i \tau A^{1 / 2}}{2}\right) R^{j-s}\right.\right.
$$$$
\left.+\left(I+\frac{i \tau A^{1 / 2}}{2}\right) \widetilde{R}^{j-s}\right]\left(f_{s}-f_{s-1}\right)
$$

From the last formula and estimates (37) it follows that

$$
\begin{aligned}
& \|A \mu\|_{H} \\
& \leq M\left\{\sum_{s=1}^{N-1}\left\|f_{s}-f_{s-1}\right\|_{H} \tau+\left\|f_{0}\right\|_{H}+\left\|A^{1 / 2} \psi\right\|_{H}+\|A \varphi\|_{H}\right\} .
\end{aligned}
$$

Applying $A^{1 / 2}$ to (48) and using Abel's formula, we get

$$
\begin{aligned}
& A^{1 / 2} \omega \\
& =T^{-1}\left\{\left[I-\left[\alpha\left(\frac{\tau}{2}\right)+\left[\alpha\left(\frac{\tau}{2}\right)+\alpha\left(\frac{3 \tau}{2}\right)\right]\right.\right.\right. \\
& \quad \times\left(\frac{R+\widetilde{R}}{2}\right)+\alpha\left(\frac{3 \tau}{2}\right)\left(\frac{R^{2}+\widetilde{R}^{2}}{2}\right) \\
& +\sum_{j=3}^{N} \alpha\left(t_{j}-\frac{\tau}{2}\right) \\
& \left.\left.\quad \times \frac{\widetilde{R}^{j}+R^{j}+R^{j-1}+\widetilde{R}^{j-1}}{2}\right]\right] \frac{\tau}{2}
\end{aligned}
$$




$$
\begin{aligned}
& \times\left\{\left[\left[\beta\left(\frac{\tau}{2}\right)-\beta\left(\frac{3 \tau}{2}\right)\right] \frac{\tau^{2}}{2} A^{1 / 2}\left(I+\frac{\tau^{2} A}{4}\right)^{-1}\right.\right. \\
& +\frac{\tau}{2} i \beta\left(\frac{3 \tau}{2}\right) \times \frac{R^{2}-\widetilde{R}^{2}}{2} \\
& +\sum_{j=3}^{N} \frac{\tau}{2} \beta\left(t_{j}-\frac{\tau}{2}\right) i \\
& \left.\times \frac{R^{j}-\widetilde{R}^{j}+\widetilde{R}^{j-1}-R^{j-1}}{2}\right] f_{0} \\
& +i \tau \frac{R-\widetilde{R}}{2}\left[\beta\left(\frac{3 \tau}{2}\right) f_{1}\right. \\
& \left.+\sum_{j=3}^{N} \beta\left(t_{j}-\frac{\tau}{2}\right) f_{j-1}\right]+A^{1 / 2} \psi \\
& -\sum_{j=3}^{N} \beta\left(t_{j}-\frac{\tau}{2}\right) \frac{\tau}{4} \\
& \times\left[\sum _ { s = 1 } ^ { j - 2 } \left[\left(I-\frac{i \tau A^{1 / 2}}{2}\right) R^{j-s}\right.\right. \\
& \left.+\left(I+\frac{i \tau A^{1 / 2}}{2}\right) \widetilde{R}^{j-s}\right]\left(f_{s}-f_{s-1}\right) \\
& -\left[\left(I-\frac{i \tau A^{1 / 2}}{2}\right) R^{j-s}\right. \\
& \left.\left.+\left(I+\frac{i \tau A^{1 / 2}}{2}\right) \widetilde{R}^{j-s}\right] f_{0}+2 f_{j-2}\right] \\
& +\sum_{j=3}^{N} \beta\left(t_{j}-\frac{\tau}{2}\right) \frac{\tau}{4} \\
& \times\left[\sum _ { s = 1 } ^ { j - 2 } \left[\left(I-\frac{i \tau A^{1 / 2}}{2}\right) R^{j-s-1}\right.\right. \\
& \left.+\left(I+\frac{i \tau A^{1 / 2}}{2}\right) \widetilde{R}^{j-s-1}\right] \\
& \times\left(f_{s}-f_{s-1}\right) \\
& +\left[\left(I-\frac{i \tau A^{1 / 2}}{2}\right) R^{j-s-1}\right. \\
& \left.\left.\left.+\left(I+\frac{i \tau A^{1 / 2}}{2}\right) \widetilde{R}^{j-s-1}\right] f_{0}-2 f_{j-2}\right]\right\} \\
& +\left[\left[\beta\left(\frac{\tau}{2}\right)-\beta\left(\frac{3 \tau}{2}\right)\right] \frac{R+\widetilde{R}}{2}-\beta\left(\frac{\tau}{2}\right)\right. \\
& +\beta\left(\frac{3 \tau}{2}\right) \frac{R^{2}+\widetilde{R}^{2}}{2}
\end{aligned}
$$

$$
\begin{aligned}
& \left.+\sum_{j=3}^{N} \beta\left(t_{j}-\frac{\tau}{2}\right) \frac{R^{j}-R^{j-1}+\widetilde{R}^{j}-\widetilde{R}^{j-1}}{2}\right] \\
& \times\left\{\left[\alpha\left(\frac{\tau}{2}\right)+\alpha\left(\frac{3 \tau}{2}\right)\right.\right. \\
& +\alpha\left(\frac{3 \tau}{2}\right) A^{1 / 2} \frac{R^{2}-\widetilde{R}^{2}}{2 i} \\
& +\sum_{j=3}^{N} \alpha\left(t_{j}-\frac{\tau}{2}\right) A^{1 / 2} \\
& \left.\times \frac{R^{j}-\widetilde{R}^{j}+R^{j-1}-\widetilde{R}^{j-1}}{2 i}\right] \frac{\tau^{2}}{4} f_{0} \\
& -\alpha\left(\frac{3 \tau}{2}\right) \frac{\tau^{2}}{4 i} A^{1 / 2}(R-\widetilde{R}) f_{1} \\
& +\frac{\tau}{2 i} A^{1 / 2}(R-\widetilde{R}) f_{j-1}+A \varphi \\
& -\sum_{j=3}^{N} \alpha\left(t_{j}-\frac{\tau}{2}\right) \frac{\tau}{4} \\
& \times\left[\sum _ { s = 1 } ^ { j - 2 } \left[\left(I-\frac{i \tau A^{1 / 2}}{2}\right) R^{j-s}\right.\right. \\
& \left.+\left(I+\frac{i \tau A^{1 / 2}}{2}\right) \widetilde{R}^{j-s}\right]\left(f_{s}-f_{s-1}\right) \\
& -\left[\left(I-\frac{i \tau A^{1 / 2}}{2}\right) R^{j-s}\right. \\
& \left.\left.+\left(I+\frac{i \tau A^{1 / 2}}{2}\right) \widetilde{R}^{j-s}\right] f_{0}+2 f_{j-2}\right] \\
& -\sum_{j=3}^{N} \alpha\left(t_{j}-\frac{\tau}{2}\right) \frac{\tau}{4} \\
& \times\left[\sum _ { s = 1 } ^ { j - 2 } \left[\left(I-\frac{i \tau A^{1 / 2}}{2}\right) R^{j-s-1}\right.\right. \\
& \left.+\left(I+\frac{i \tau A^{1 / 2}}{2}\right) \widetilde{R}^{j-s-1}\right]\left(f_{s}-f_{s-1}\right) \\
& -\left[\left(I-\frac{i \tau A^{1 / 2}}{2}\right) R^{j-s-1}\right. \\
& \left.\left.\left.\left.+\left(I+\frac{i \tau A^{1 / 2}}{2}\right) \widetilde{R}^{j-s-1}\right] f_{0}+2 f_{j-2}\right]\right\}\right\} .
\end{aligned}
$$


Using the triangle inequality and estimates (37), we obtain

$$
\begin{aligned}
& \left\|A^{1 / 2} \omega\right\|_{H} \\
& \quad \leq M\left\{\sum_{s=1}^{N-1}\left\|f_{s}-f_{s-1}\right\|_{H} \tau+\left\|f_{0}\right\|_{H}+\left\|A^{1 / 2} \psi\right\|_{H}+\|A \varphi\|_{H}\right\} .
\end{aligned}
$$

As a result, estimate (44) follows from estimates (50), (59), and (61). Theorem 6 is proved.

\section{Application}

The discretization of hyperbolic equation (2) with Neumann and integral or nonclassical and integral boundary conditions is carried out in two steps. First, let us define the grid sets

$$
\begin{gathered}
\widetilde{\Omega}_{h}=\left\{x=x_{r}=\left(h_{1} r_{1}, \ldots, h_{m} r_{m}\right), r=\left(r_{1}, \ldots, r_{m}\right),\right. \\
\left.0 \leq r_{j} \leq N_{j}, h_{j} N_{j}=1, j=1, \ldots, m\right\}, \\
\Omega_{h}=\widetilde{\Omega}_{h} \cap \Omega, \quad S_{h}=\widetilde{\Omega}_{h} \cap S .
\end{gathered}
$$

We introduce the Banach space $L_{2 h}=L_{2}\left(\widetilde{\Omega}_{h}\right)$ of the grid functions

$$
\varphi^{h}(x)=\left\{\varphi\left(h_{1} r_{1}, \ldots, h_{m} r_{m}\right)\right\}
$$

defined on $\widetilde{\Omega}_{h}$, equipped with the norm

$$
\left\|\varphi^{h}\right\|_{L_{2}\left(\widetilde{\Omega}_{h}\right)}=\left(\sum_{x \in \overline{\Omega_{h}}}\left|\varphi^{h}(x)\right|^{2} h_{1} \cdots h_{m}\right)^{1 / 2}
$$

To the differential operator $A^{x}$ generated by (2), we assign the difference operator $A_{h}^{x}$ by the formula

$$
A_{h}^{x} u_{x}^{h}=-\sum_{r=1}^{m}\left(a_{r}(x) u_{\bar{x}_{r}}^{h}\right)_{x_{r} r_{j}}+\sigma u^{h}(x)
$$

acting in the space of grid functions $u^{h}(x)$, satisfying the condition $D^{h} u^{h}(x)=0$ for all $x \in S_{h}$ or $u^{h}(x)=0, x \in S_{1 h}$ and $D^{h} u^{h}(x)=0, x \in S_{2 h}, S_{h}=S_{1 h} \cup S_{2 h} . D^{h} u^{h}$ is the approximation of $(\partial u / \partial \vec{n})$. It is known that $A_{h}^{x}$ is a self-adjoint positive definite operator in $L_{2}\left(\widetilde{\Omega}_{h}\right)$. With the help of $A_{h}^{x}$ we arrive at the nonlocal boundary value problem

$$
\begin{gathered}
\frac{d^{2} v^{h}(t, x)}{d t^{2}}+A_{h}^{x} v^{h}(t, x)=f^{h}(t, x), \quad 0<t<1, \quad x \in \widetilde{\Omega}_{h}, \\
v^{h}(0, x)=\int_{0}^{1} \alpha(\rho) v^{h}(\rho, x) d \rho+\varphi^{h}(x), \quad x \in \widetilde{\Omega}_{h}, \\
\frac{d v^{h}(0, x)}{d t}=\int_{0}^{1} \beta(\rho) \frac{d v^{h}(\rho, x)}{d t} d \rho+\psi^{h}(x), \quad x \in \widetilde{\Omega}_{h}
\end{gathered}
$$

for an infinite system of ordinary differential equations. Second, we replace problem (66) by the difference scheme

$$
\begin{gathered}
\frac{u_{k+1}^{h}(x)-2 u_{k}^{h}(x)+u_{k-1}^{h}(x)}{\tau^{2}}+A_{h}^{x} u_{k+1}^{h}(x)=f_{k+1}^{h}(x), \\
f_{k+1}^{h}(x)=f^{h}\left(t_{k+1}, x\right), \quad t_{k+1}=(k+1) \tau, \\
1 \leq k \leq N-1, \quad N \tau=1, \quad x \in \widetilde{\Omega}_{h}, \\
u_{0}^{h}(x)=\sum_{m=1}^{N} \alpha\left(t_{m}\right) u_{m}^{h}(x) \tau+\varphi^{h}(x), \quad x \in \widetilde{\Omega}_{h}, \\
\left(I+\tau^{2} A_{h}^{x}\right)\left(u_{1}^{h}(x)-u_{0}^{h}(x)\right) \tau^{-1} \\
=\sum_{j=1}^{N} \beta\left(\rho_{j}\right)\left[u_{j}^{h}(x)-u_{j-1}^{h}(x)\right]+\psi^{h}(x), \quad x \in \widetilde{\Omega}_{h}
\end{gathered}
$$

of the first order accuracy in $t$. For the stability of first order of accuracy difference scheme, the following theorem is presented.

Theorem 7. Let $\tau$ and $h$ be sufficiently small numbers. Then, the solutions of difference scheme (67) satisfy the following stability estimates:

$$
\begin{aligned}
& \max _{0 \leq k \leq N}\left\|u_{k}^{h}\right\|_{L_{2 h}}+\max _{1 \leq k \leq N}\left\|\tau^{-1}\left(u_{k}^{h}-u_{k-1}^{h}\right)\right\|_{L_{2 h}}+\max _{0 \leq k \leq N}\left\|\left(u_{k}^{h}\right)_{x}\right\|_{L_{2 h}} \\
& \leq M_{1}\left[\max _{1 \leq k \leq N-1}\left\|f_{k}^{h}\right\|_{L_{2 h}}+\left\|\varphi_{\bar{x}}^{h}\right\|_{L_{2 h}}+\left\|\psi^{h}\right\|_{L_{2 h}}\right] \\
& \max _{1 \leq k \leq N-1}\left\|\tau^{-2}\left(u_{k+1}^{h}-2 u_{k}^{h}+u_{k-1}^{h}\right)\right\|_{L_{2 h}}+\max _{1 \leq k \leq N}\left\|\left(u_{\bar{x}}^{k}\right)_{x}\right\|_{L_{2 h}} \\
& \leq M_{1}\left[\left\|f_{1}^{h}\right\|_{L_{2 h}}+\max _{1 \leq k \leq N-1}\left\|\tau^{-1}\left(f_{k}^{h}-f_{k-1}^{h}\right)\right\|_{L_{2 h}}\right. \\
& \left.+\left\|\left(\varphi_{\bar{x}}^{h}\right)_{x}\right\|_{L_{2 h}}+\left\|\psi \frac{h}{x}\right\|_{L_{2 h}}\right] .
\end{aligned}
$$

Here, $M_{1}$ is independent of $\tau, h, \varphi^{h}(x), \psi^{h}(x)$, and $f_{k}^{h}(x), 1 \leq$ $k<N$.

The proof of Theorem 7 is based on the symmetry property of difference operator $A_{h}^{x}$ defined by formula (65) and on the following theorem on coercivity inequality of the elliptic difference problem.

Theorem 8. For the solutions of the elliptic difference problem

$$
\begin{gathered}
A_{h}^{x} u^{h}(x)=\omega^{h}(x), \quad x \in \Omega_{h}, \quad D^{h} u^{h}(x)=0, \quad x \in S_{h} \\
\text { or } \quad u^{h}(x)=0, \quad x \in S_{1 h}, \quad D^{h} u^{h}(x)=0, \quad x \in S_{2 h}
\end{gathered}
$$


the following coercivity inequality holds [23]:

$$
\sum_{r=1}^{m}\left\|u_{x_{r} \bar{x}_{r} r_{j}}^{h}\right\|_{L_{2 h}} \leq M\left\|\omega^{h}\right\|_{L_{2 h}} .
$$

In addition, the second order of accuracy difference scheme

$$
\begin{gathered}
\tau^{-2}\left[u_{k+1}^{h}(x)-2 u_{k}^{h}(x)+u_{k-1}^{h}(x)\right] \\
+\frac{1}{2} A_{h}^{x} u_{k}^{h}(x)+\frac{1}{4} A_{h}^{x}\left[u_{k+1}^{h}(x)+u_{k-1}^{h}(x)\right]=f_{k}^{h}(x), \\
x \in \widetilde{\Omega}_{h}, \quad f_{k}^{h}=f_{k}^{h}\left(t_{k}, x\right), \\
t_{k}=k \tau, \quad 1 \leq k \leq N-1, \quad N \tau=1, \\
u_{0}^{h}(x)=\sum_{j=1}^{N} \alpha\left(\rho_{j}-\frac{\tau}{2}\right)\left[u_{j}^{h}(x)+u_{j-1}^{h}(x)\right] \frac{\tau}{2} \\
+\varphi^{h}(x), \quad x \in \widetilde{\Omega}_{h}, \\
\left(I+\frac{\tau^{2} A_{h}^{x}}{2}\right) \tau^{-1}\left[u_{1}^{h}(x)-u_{0}^{h}(x)\right] \\
f_{0}^{h}=f^{h}(0, x), \\
-\frac{\tau}{2}\left[f_{0}^{h}(x)-A_{h}^{x} u_{0}^{h}(x)\right] \\
=\sum_{j=1}^{N} \beta\left(\rho_{j}-\frac{\tau}{2}\right)\left[u_{j}^{h}(x)-u_{j-1}^{h}(x)\right]+\psi^{h}(x),
\end{gathered}
$$

for approximately solving hyperbolic equation (2) with nonlocal integral and Neumann or nonclassical conditions is presented. The following theorem on the stability of (71) is obtained.

Theorem 9. Let $\tau$ and $h$ be sufficiently small numbers. Then, the solution of difference scheme (71) satisfies the following stability estimates:

$$
\begin{gathered}
\max _{0 \leq k \leq N}\left\|u_{k}^{h}\right\|_{L_{2 h}}+\max _{1 \leq k \leq N}\left\|\tau^{-1}\left(u_{k}^{h}-u_{k-1}^{h}\right)\right\|_{L_{2 h}}+\max _{0 \leq k \leq N}\left\|\left(u_{k}^{h}\right)_{x}\right\|_{L_{2 h}} \\
\leq M_{2}\left[\left\|f_{0}^{h}\right\|_{L_{2 h}}+\max _{1 \leq k \leq N}\left\|\left(f_{k}^{h}-f_{k-1}^{h}\right) \tau^{-1}\right\|_{L_{2 h}}\right. \\
\left.+\left\|\varphi_{\frac{h}{x}}\right\|_{L_{2 h}}+\left\|\psi^{h}\right\|_{L_{2 h}}\right],
\end{gathered}
$$

$$
\begin{gathered}
\max _{1 \leq k \leq N-1}\left\|\tau^{-2}\left(u_{k+1}^{h}-2 u_{k}^{h}+u_{k-1}^{h}\right)\right\|_{L_{2 h}} \\
+\max _{1 \leq k \leq N}\left\|\frac{\left(u_{\bar{x}}^{k}\right)_{x}+\left(u_{\bar{x}}^{k-1}\right)_{x}}{2}\right\|_{L_{2 h}} \\
\leq M_{2}\left[\left\|f_{0}^{h}\right\|_{L_{2 h}}+\left\|\tau^{-1}\left(f_{1}^{h}-f_{0}^{h}\right)\right\|_{L_{2 h}}\right. \\
+\max _{1 \leq k \leq N-1}\left\|\tau^{-1}\left(f_{k}^{h}-f_{k-1}^{h}\right)\right\|_{L_{2 h}} \\
\left.+\left\|\left(\varphi_{\bar{x}}^{h}\right)_{x}\right\|_{L_{2 h}}+\left\|\psi_{\bar{x}}^{h}\right\|_{L_{2 h}}\right] .
\end{gathered}
$$

Here, $M_{2}$ does not depend on $\tau, h, \varphi^{h}(x)$, and $f_{k}^{h}, 0 \leq k<N-1$.

The proof of Theorem 9 is based on the symmetry property of difference operator $A_{h}^{x}$ defined by formula (65) and on Theorem 8 on coercivity inequality of elliptic difference problem (69).

\section{Numerical Examples}

In this section, we apply finite difference schemes (67) and (71) to three examples which are one-dimensional hyperbolic equation with nonlocal and nonclassical conditions.

Example 10. The nonlocal boundary value problem

$$
\begin{gathered}
\frac{\partial^{2} u(t, x)}{\partial t^{2}}-(1+x) \frac{\partial^{2} u(t, x)}{\partial x^{2}}-u_{x}(t, x)+u(t, x) \\
=f(t, x), \\
f(t, x)=[(x+2) \cos x-\sin x]\left(e^{t}-1-t\right)+e^{t} \cos x, \\
0<t<1, \quad 0<x<\pi, \\
u(0, x)=\int_{0}^{1} e^{-s} u(s, x) d s+\varphi(x), \\
\varphi(x)=\left(1-3 e^{-1}\right) \cos x, \\
u_{t}(0, x)=\int_{0}^{1} e^{-s} u_{s}(s, x) d s+\psi(x), \\
\psi(x)=-e^{-1} \cos x, \\
0 \leq x \leq \pi, \\
u_{x}(t, 0)=u_{x}(t, \pi)=0, \quad 0 \leq t \leq 1,
\end{gathered}
$$

for one-dimensional hyperbolic equation with variable coefficients is considered. The exact solution of this problem is

$$
u(t, x)=\left(e^{t}-1-t\right) \cos x .
$$

For the approximate solution of the problem (73), we apply finite difference schemes (67) and (71). 
First, we obtain the first order of accuracy difference scheme

$$
\begin{gathered}
\frac{u_{n}^{k+1}-2 u_{n}^{k}+u_{n}^{k-1}}{\tau^{2}}-\left(1+x_{n}\right) \frac{u_{n+1}^{k+1}-2 u_{n}^{k+1}+u_{n-1}^{k+1}}{h^{2}} \\
-\frac{u_{n+1}^{k+1}-u_{n-1}^{k+1}}{2 h}+u_{n}^{k+1}=f\left(t_{k+1}, x_{n}\right), \\
f\left(t_{k+1}, x_{n}\right)=\left[\left(x_{n}+2\right) \cos x_{n}-\sin x_{n}\right] \times\left(e^{t_{k+1}}-1-t_{k+1}\right) \\
+e^{t_{k+1}} \cos x_{n}, \quad N \tau=1, x_{n}=n h, \\
1 \leq n \leq M-1, \quad M h=\pi, \\
u_{n}^{0}-\sum_{k=1}^{N} e^{-k \tau} \tau u_{n}^{k}=\varphi\left(x_{n}\right), \quad t_{k}=k \tau, \quad 1 \leq k \leq N-1, \\
\varphi\left(x_{n}\right)=\left(1-3 e^{-1}\right) \cos x_{n}, \quad 0 \leq n \leq M, \\
\psi\left(x_{n}\right)=-e^{-1} \cos x_{n}, \quad 0 \leq n \leq M-1, \\
u_{0}^{k}=u_{1}^{k}, \quad u_{M}^{k}=u_{M-1}^{k}, \quad 0 \leq k \leq N .
\end{gathered}
$$

The system can be written in the matrix form

$$
\begin{gathered}
A_{n} u_{n+1}+B_{n} u_{n}+C_{n} u_{n-1}=D \varphi_{n}, \quad 1 \leq n \leq M-1, \\
u_{0}=u_{1}, \quad u_{M}=u_{M-1} .
\end{gathered}
$$

Here,

$$
\begin{aligned}
& A_{n}=\left[\begin{array}{ccccccc}
0 & 0 & 0 & 0 & \cdots & 0 & 0 \\
0 & 0 & a_{n} & 0 & \cdots & 0 & 0 \\
0 & 0 & 0 & a_{n} & \cdots & 0 & 0 \\
\vdots & \vdots & \vdots & \vdots & \ddots & \vdots & \vdots \\
0 & 0 & 0 & 0 & \cdots & a_{n} & 0 \\
0 & 0 & 0 & 0 & \cdots & 0 & a_{n} \\
0 & 0 & 0 & 0 & \cdots & 0 & 0
\end{array}\right]_{(N+1) \times(N+1)} \\
& B_{n}=\left[\begin{array}{cccccc}
1 & -\tau e^{-\tau} & -\tau e^{-2 \tau} & \cdots & -\tau e^{-(N-1) \tau} & -\tau e^{-N \tau} \\
b & c & d_{n} & \cdots & 0 & 0 \\
0 & b & c & \cdots & 0 & 0 \\
0 & 0 & b & \cdots & 0 & 0 \\
\vdots & \vdots & \vdots & \ddots & \vdots & \vdots \\
0 & 0 & 0 & \cdots & d_{n} & 0 \\
0 & 0 & 0 & \cdots & c & d_{n} \\
-1+e^{-\tau} & a t & a p & \cdots & a n & -e^{-N \tau}
\end{array}\right]_{(N+1) \times(N+1)}
\end{aligned}
$$

$$
\begin{aligned}
& C_{n}=\left[\begin{array}{ccccccc}
0 & 0 & 0 & 0 & \cdots & 0 & 0 \\
0 & 0 & e_{n} & 0 & \cdots & 0 & 0 \\
0 & 0 & 0 & e_{n} & \cdots & 0 & 0 \\
\vdots & \vdots & \vdots & \vdots & \ddots & \vdots & \vdots \\
0 & 0 & 0 & 0 & \cdots & e_{n} & 0 \\
0 & 0 & 0 & 0 & \cdots & 0 & e_{n} \\
0 & 0 & 0 & 0 & \cdots & 0 & 0
\end{array}\right]_{(N+1) \times(N+1)} \\
& a_{n}=\frac{-\left(1+x_{n}\right)}{h^{2}}-\frac{1}{2 h}, \quad b=\frac{1}{\tau^{2}}, \quad c=\frac{-2}{\tau^{2}}, \\
& d_{n}=\frac{1}{\tau^{2}}+\frac{2\left(1+x_{n}\right)}{h^{2}}+1, \quad e_{n}=\frac{-\left(1+x_{n}\right)}{h^{2}}+\frac{1}{2 h}, \\
& \text { at }=1-e^{-\tau}+e^{-2 \tau}, \quad \text { ap }=-e^{-2 \tau}+e^{-3 \tau} \text {, } \\
& a n=-e^{-(N-1) \tau}+e^{-N \tau} \text {, } \\
& f_{n}=\left[\begin{array}{c}
f_{n}^{0} \\
f_{n}^{1} \\
f_{n}^{2} \\
\vdots \\
f_{n}^{N}
\end{array}\right]_{(N+1) \times 1}, \\
& f_{n}^{k+1}=f\left(t_{k+1}, x_{n}\right)=\left[\left(x_{n}+2\right) \cos x_{n}-\sin x_{n}\right] \\
& \times\left(e^{t_{k}}-1-t_{k}\right)+e^{t_{k}} \cos x_{n}, \quad 1 \leq k \leq N-1, \\
& f_{n}^{0}=\left(1-3 e^{-1}\right) \cos x_{n}, \quad 0 \leq n \leq M, \\
& f_{n}^{N}=-e^{-1} \cos x_{n}, \quad 0 \leq n \leq M,
\end{aligned}
$$

and $D=I_{N+1}$ is the identity matrix.

Consider

$$
U_{s}=\left[\begin{array}{c}
u_{s}^{0} \\
u_{s}^{1} \\
u_{s}^{2} \\
\vdots \\
u_{s}^{N}
\end{array}\right]_{(N+1) \times 1}, \quad s=n-1, n, n+1 .
$$

This type system was used by [24] for difference equations. For the solution of matrix equation (76), we will use modified Gauss elimination method. We seek a solution of the matrix equation by the following form:

$$
u_{n}=\alpha_{n+1} u_{n+1}+\beta_{n+1}, \quad n=M-1, \ldots, 2,1,
$$


where $u_{M}=\left(I-\alpha_{M}\right)^{-1} \beta_{M}, \alpha_{j}(j=1, \ldots, M-1)$ are $(N+$ $1) \times(N+1)$ square matrices and $\beta_{j}(j=1, \ldots, M-1)$ are $(N+1) \times 1$ column matrices. $\alpha_{1}$ is identity and $\beta_{1}$ is zero matrices, and

$$
\begin{gathered}
\alpha_{n+1}=-\left(B_{n}+C_{n} \alpha_{n}\right)^{-1} A_{n}, \\
\beta_{n+1}=\left(B_{n}+C_{n} \alpha_{n}\right)^{-1}\left(D_{n} \varphi_{n}-C_{n} \beta_{n}\right), \\
n=1,2,3, \ldots, M-1 .
\end{gathered}
$$

Second, applying formulas

$$
\begin{aligned}
& \frac{2 u(0)-5 u(h)+4 u(2 h)-u(3 h)}{h^{2}}-u^{\prime \prime}(0)=O\left(h^{2}\right), \\
& \frac{2 u(\pi)-5 u(\pi-h)+4 u(\pi-2 h)-u(\pi-3 h)}{h^{2}}-u^{\prime \prime}(\pi) \\
& \quad=O\left(h^{2}\right)
\end{aligned}
$$

and using the second order of accuracy implicit difference scheme (71), we get second order of accuracy difference scheme

$$
\begin{aligned}
& \frac{u_{n}^{k+1}-2 u_{n}^{k}+u_{n-1}^{k-1}}{\tau^{2}}-\left(1+x_{n}\right) \\
& \times\left[\frac{u_{n+1}^{k-1}-2 u_{n}^{k-1}+u_{n-1}^{k-1}}{4 h^{2}}-\frac{u_{n+1}^{k}-2 u_{n}^{k}+u_{n-1}^{k}}{2 h^{2}}\right. \\
& \left.\quad-\frac{u_{n+1}^{k+1}-2 u_{n}^{k+1}+u_{n-1}^{k+1}}{4 h^{2}}\right] \\
& -\left[\frac{u_{n+1}^{k-1}-u_{n-1}^{k-1}}{8 h}+\frac{u_{n+1}^{k}-u_{n-1}^{k}}{4 h}+\frac{u_{n+1}^{k+1}-u_{n-1}^{k+1}}{8 h}\right] \\
& +\frac{1}{2} u_{n}^{k}+\frac{u_{n}^{k+1}+u_{n}^{k-1}}{4}=f_{n}^{k},
\end{aligned}
$$

$$
\begin{aligned}
& f_{n}^{k}=\left[\left(x_{n}+2\right) \cos x_{n}+\sin x_{n}\right]\left(e^{t_{k}}-1-t_{k}\right)+e^{t_{k}} \cos x_{n}, \\
& M h=\pi, \quad x_{n}=n h, \quad 1 \leq n \leq M-1, \quad N \tau=1, \\
& t_{k}=k \tau, \quad 1 \leq k \leq N-1, \\
& u_{n}^{0}=\sum_{k=1}^{N} \frac{\tau}{2}\left[e^{-k \tau} u_{n}^{k}+e^{-(k-1) \tau} u_{n}^{k-1}\right]+\varphi\left(x_{n}\right) \text {, } \\
& 0 \leq n \leq M, \\
& \varphi\left(x_{n}\right)=\left(1-3 e^{-1}\right) \cos x_{n}, \quad 0 \leq n \leq M, \\
& \frac{u_{n}^{1}-u_{n}^{0}}{\tau}-\frac{\tau}{4}\left(1+x_{n}\right) \\
& \times\left[\frac{u_{n+1}^{1}-2 u_{n}^{1}+u_{n-1}^{1}}{h^{2}}+\frac{u_{n+1}^{0}-2 u_{n}^{0}+u_{n-1}^{0}}{h^{2}}\right] \\
& +\frac{\tau}{2}\left[-\frac{u_{n+1}^{1}-u_{n-1}^{1}}{4 h}-\frac{u_{n+1}^{0}-u_{n-1}^{0}}{4 h}\right] \\
& +\frac{\tau}{4}\left[u_{n}^{0}+u_{n}^{1}\right]-\frac{\tau}{4} f_{n}^{0} \\
& =\sum_{k=1}^{N} e^{-k \tau+(\tau / 2)}\left[u_{n}^{k}+u_{n}^{k-1}\right]+\psi\left(x_{n}\right), \quad 1 \leq n \leq M-1, \\
& \psi\left(x_{n}\right)=-e^{-1} \cos x_{n}, \quad 0 \leq n \leq M, \\
& u_{1}^{0}-u_{0}^{0}=\frac{h^{2}}{2}\left[\frac{2 u_{0}^{0}-5 u_{0}^{1}+4 u_{0}^{2}-u_{0}^{3}}{\tau^{2}}+u_{0}^{0}-\varphi_{0}^{0}\right] \\
& u_{1}^{N}-u_{0}^{N} \\
& =\frac{h^{2}}{2}\left[\frac{2 u_{0}^{N}-5 u_{0}^{N-1}+4 u_{0}^{N-2}-u_{0}^{N-3}}{\tau^{2}}+u_{0}^{N}-\varphi_{0}^{N}\right] \\
& u_{1}^{k}-u_{0}^{k}=\frac{h^{2}}{2}\left[\frac{u_{0}^{k+1}-2 u_{0}^{k}+u_{0}^{k-1}}{h^{2}}+u_{0}^{k}-\varphi_{0}^{k}\right] \text {, } \\
& 1 \leq k \leq N-1 \text {, }
\end{aligned}
$$

for the approximate solutions of nonlocal boundary value problem (73). We have again $(N+1) \times(N+1)$ system of linear equations. We can write the system as a matrix equation (76). Here,

$$
E_{n}=\left[\begin{array}{cccccccc}
0 & 0 & 0 & 0 & \cdots & 0 & 0 & 0 \\
a_{n} & 2 a_{n} & a_{n} & 0 & \cdots & 0 & 0 & 0 \\
0 & a_{n} & 2 a_{n} & a_{n} & \cdots & 0 & 0 & 0 \\
\vdots & \vdots & \vdots & \vdots & \ddots & \vdots & \vdots & \vdots \\
0 & 0 & 0 & 0 & \cdots & a_{n} & 2 a_{n} & a_{n} \\
w_{n} & w_{n} & 0 & 0 & \cdots & 0 & 0 & 0
\end{array}\right]_{(N+1) \times(N+1)}
$$




$$
\begin{aligned}
& F_{n}=\left[\begin{array}{ccccccc}
1-\frac{\tau}{2} & -\tau e^{-\tau} & -\tau e^{-2 \tau} & -\tau e^{-3 \tau} & \cdots & -\tau e^{-(N-1) \tau} & -\frac{\tau}{2} e^{-N \tau} \\
b_{n} & d_{n} & b_{n} & 0 & \cdots & 0 & 0 \\
0 & b_{n} & d_{n} & b_{n} & \cdots & 0 & 0 \\
\vdots & \vdots & \vdots & \vdots & \ddots & \vdots & \vdots \\
0 & 0 & 0 & 0 & \cdots & b_{n} & 0 \\
0 & 0 & 0 & 0 & \cdots & d_{n} & b_{n} \\
y_{n} & t_{n} & \lambda e^{-3 \tau / 2} & \lambda e^{-5 \tau / 2} & \cdots & \lambda e^{-(2 N-3) \tau / 2} & -e^{-N \tau+(\tau / 2)}
\end{array}\right]_{(N+1) \times(N+1)} \\
& G_{n}=\left[\begin{array}{cccccccc}
0 & 0 & 0 & 0 & \cdots & 0 & 0 & 0 \\
c_{n} & 2 c_{n} & c_{n} & 0 & \cdots & 0 & 0 & 0 \\
0 & c_{n} & 2 c_{n} & c_{n} & \cdots & 0 & 0 & 0 \\
\vdots & \vdots & \vdots & \vdots & \ddots & \vdots & \vdots & \vdots \\
0 & 0 & 0 & 0 & \cdots & c_{n} & 2 c_{n} & c_{n} \\
z_{n} & z_{n} & 0 & 0 & \cdots & 0 & 0 & 0
\end{array}\right]_{(N+1) \times(N+1)} \\
& a_{n}=\frac{-\left(1+x_{n}\right)}{4 h^{2}}-\frac{1}{8 h}, \quad b_{n}=\frac{1}{\tau^{2}}+\frac{1+x_{n}}{2 h^{2}}+\frac{1}{4}, \\
& c_{n}=\frac{-\left(1+x_{n}\right)}{4 h^{2}}+\frac{1}{8 h}, \quad d_{n}=\frac{-2}{\tau^{2}}+\frac{1+x_{n}}{h^{2}}+\frac{1}{2}, \\
& y_{n}=-\frac{1}{\tau}+\frac{\tau}{4}+\frac{\left(1+x_{n}\right) \tau}{2 h^{2}}+e^{-\tau / 2} \text {, } \\
& t_{n}=\frac{1}{\tau}+\frac{\tau}{4}+\frac{\left(1+x_{n}\right) \tau}{2 h^{2}}+\left(e^{-\tau}-1\right) e^{-\tau / 2}, \\
& w_{n}=-\frac{\tau^{2}}{4 h}-\frac{\left(1+x_{n}\right) \tau^{2}}{2 h^{2}}, \quad z_{n}=\frac{\tau^{2}}{4 h}-\frac{\left(1+x_{n}\right) \tau^{2}}{2 h^{2}}, \quad \lambda=\left(e^{-\tau}-1\right) \\
& f_{n}=\left[\begin{array}{c}
f_{n}^{0} \\
f_{n}^{1} \\
\vdots \\
f_{n}^{N}
\end{array}\right]_{(N+1) \times 1}, \\
& f_{n}^{k}=f\left(t_{k}, x_{n}\right)=\left[\left(x_{n}+2\right) \cos x_{n}-\sin x_{n}\right] \\
& \times\left(e^{t_{k}}-1-t_{k}\right)+e^{t_{k}} \cos x_{n}, \quad 1 \leq k \leq N-1, \\
& f_{n}^{0}=\left(1-3 e^{-1}\right) \cos x_{n}, \quad 0 \leq n \leq M, \\
& f_{n}^{N}=-e^{-1} \cos x_{n}, \quad 0 \leq n \leq M, \\
& T=\left[\begin{array}{cccccccccccc}
\lambda_{1} & \lambda_{2} & \lambda_{3} & \lambda_{4} & 0 & 0 & \cdots & 0 & 0 & 0 & 0 & 0 \\
a & b & a & 0 & 0 & 0 & \cdots & 0 & 0 & 0 & 0 & 0 \\
0 & a & b & a & 0 & 0 & \cdots & 0 & 0 & 0 & 0 & 0 \\
\vdots & \vdots & \vdots & \vdots & \vdots & \vdots & \ddots & \vdots & \vdots & \vdots & \vdots & \vdots \\
0 & 0 & 0 & 0 & 0 & 0 & \cdots & 0 & a & b & a & 0 \\
0 & 0 & 0 & 0 & 0 & 0 & \cdots & 0 & 0 & a & b & a \\
0 & 0 & 0 & 0 & 0 & 0 & \cdots & 0 & \lambda_{4} & \lambda_{3} & \lambda_{2} & \lambda_{1}
\end{array}\right]_{(N+1) \times(N+1)}
\end{aligned}
$$




$$
\begin{gathered}
a=\frac{-h}{2 \tau^{2}}, \quad b=\frac{-1}{h}+\frac{h}{\tau^{2}}-\frac{h}{2}, \\
\lambda_{1}=\frac{-1}{h}-\frac{h}{2}\left(\frac{2}{\tau^{2}}+1\right), \quad \lambda_{2}=\frac{5}{2} \frac{h}{\tau^{2}}, \quad \lambda_{3}=-2 \frac{h}{\tau^{2}}, \quad \lambda_{4}=\frac{h}{2 \tau^{2}}, \\
D=I_{N+1}, \quad U_{s}=\left[\begin{array}{c}
u_{s}^{0} \\
u_{s}^{1} \\
u_{s}^{2} \\
\vdots \\
u_{s}^{N}
\end{array}\right]_{(N+1) \times 1} \quad, \quad s=n-1, n, n+1 .
\end{gathered}
$$

For the solution of the matrix equation (76), we used the same algorithm as in the first order of accuracy difference scheme. Here, $u_{M}=\left(\alpha_{M-1} \alpha_{M}-4 \alpha_{M}+3 I\right)^{-1}\left(4 \beta_{M}-\alpha_{M-1} \beta_{M}-\beta_{M-1}\right)$,

$$
\alpha_{1}=T^{-1}\left(\frac{-1}{2} I\right), \quad \beta_{1}=T^{-1} \varphi_{0}^{k}, \quad 0 \leq k \leq N
$$

Example 11. The nonlocal boundary value problem

$$
\begin{gathered}
\frac{\partial^{2} u(t, x)}{\partial t^{2}}-(1+x) \frac{\partial^{2} u(t, x)}{\partial x^{2}}-u_{x}(t, x)+u(t, x) \\
=f(t, x), \\
f(t, x)=[(x+2) \cos x+\sin x-1]\left(e^{t}-1-t\right) \\
+e^{t}(\cos x-1), \quad 0<t<1,0<x<\pi, \\
u(0, x)=\int_{0}^{1} e^{-s} u(s, x) d s+\varphi(x), \\
\varphi(x)=\left(1-3 e^{-1}\right)(\cos x-1), \\
u_{t}(0, x)=\int_{0}^{1} e^{-s} u_{s}(s, x) d s+\psi(x), \\
\psi(x)=-e^{-1}(\cos x-1), \quad 0 \leq x \leq \pi, \\
u(t, 0)=u_{x}(t, \pi)=0, \quad 0 \leq t \leq 1,
\end{gathered}
$$

is considered. Here, we use the same procedure as in the first example. The exact solution of this problem is

$$
u(t, x)=\left(e^{t}-1-t\right)(\cos x-1) .
$$

Using the same manner, we can construct first order of accuracy difference scheme and it can be written in the matrix form

$$
\begin{gathered}
A_{n} u_{n+1}+B_{n} u_{n}+C_{n} u_{n-1}=D f_{n}, \quad 1 \leq n \leq M-1, \\
u_{0}=\overrightarrow{0}, \quad u_{M}=u_{M-1} .
\end{gathered}
$$

Here, matrices $A_{n}, B_{n}, C_{n}$, and $D$ are given in the previous example, and

$$
\begin{gathered}
f_{n}=\left[\begin{array}{c}
f_{n}^{0} \\
f_{n}^{1} \\
f_{n}^{2} \\
\vdots \\
f_{n}^{N}
\end{array}\right]_{(N+1) \times 1}, \\
f_{n}^{k+1}=f\left(t_{k+1}, x_{n}\right)=[(x+2) \cos x+\sin x-1] \\
\times\left(e^{t}-1-t\right)+e^{t}(\cos x-1), \quad 1 \leq k \leq N-1, \\
f_{n}^{0}=\left(1-3 e^{-1}\right)(\cos x-1), \quad 0 \leq n \leq M, \\
f_{n}^{N}=-e^{-1}(\cos x-1), \quad 0 \leq n \leq M .
\end{gathered}
$$

For the solution of matrix equation (87), we will use modified Gauss elimination method. We seek a solution of the matrix equation by the following form:

$$
u_{n}=\alpha_{n+1} u_{n+1}+\beta_{n+1}, \quad n=M-1, \ldots, 2,1,
$$

where $u_{M}=\left(I-\alpha_{M}\right)^{-1} \beta_{M}, \alpha_{j}(j=1, \ldots, M-1)$ are $(N+$ $1) \times(N+1)$ square matrices and $\beta_{j}(j=1, \ldots, M-1)$ are $(N+1) \times 1$ column matrices. $\alpha_{1}$ and $\beta_{1}$ are zero matrices, and

$$
\begin{gathered}
\alpha_{n+1}=-\left(B_{n}+C_{n} \alpha_{n}\right)^{-1} A_{n}, \\
\beta_{n+1}=\left(B_{n}+C_{n} \alpha_{n}\right)^{-1}\left(D_{n} \varphi_{n}-C_{n} \beta_{n}\right), \\
n=1,2,3, \ldots M-1 .
\end{gathered}
$$

By using the second order of accuracy implicit difference scheme (71), we can write the matrix form

$$
\begin{gathered}
E_{n} u_{n+1}+F_{n} u_{n}+G_{n} u_{n-1}=D f_{n}, \quad 1 \leq n \leq M-1, \\
u_{0}=\overrightarrow{0}, \quad u_{M-2}-4 u_{M-1}+3 u_{M}=0 .
\end{gathered}
$$


Here, matrices $E_{n}, F_{n}, G_{n}$, and $D$ are given in the previous example, and also $f_{n}$ is given in the first order accuracy difference scheme. For the solution of the matrix equation (91), we used the same algorithm as in the first order of accuracy difference scheme, where $u_{M}=\left(\alpha_{M-1} \alpha_{M}-4 \alpha_{M}+\right.$ $3 I)^{-1}\left(4 \beta_{M}-\alpha_{M-1} \beta_{M}-\beta_{M-1}\right), \alpha_{1}$ and $\beta_{1}$ are zero matrices.

Example 12. In this example, the nonlocal boundary value problem

$$
\begin{gathered}
\frac{\partial^{2} u(t, x)}{\partial t^{2}}-(1+x) \frac{\partial^{2} u(t, x)}{\partial x^{2}}-u_{x}(t, x)+u(t, x) \\
=f(t, x) \\
f(t, x)=[(x+2) \cos x+\sin x+1]\left(e^{t}-1-t\right) \\
+e^{t}(\cos x+1), \quad 0<t<1,0<x<\pi, \\
u(0, x)=\int_{0}^{1} e^{-s} u(s, x) d s+\varphi(x), \\
\varphi(x)=\left(1-3 e^{-1}\right)(\cos x+1), \\
u_{t}(0, x)=\int_{0}^{1} e^{-s} u_{s}(s, x) d s+\psi(x), \\
\psi(x)=-e^{-1}(\cos x+1), \\
u_{x}(t, 0)=u(t, \pi)=0, \quad 0 \leq t \leq 1,0 \leq x \leq \pi
\end{gathered}
$$

for one-dimensional hyperbolic equation is considered. The exact solution of this problem is

$$
u(t, x)=\left(e^{t}-1-t\right)(\cos x+1) .
$$

First, we use the first order of accuracy implicit difference scheme (67) for the approximate solutions of nonlocal boundary value problem (92) and we obtain the matrix equation

$$
\begin{gathered}
A_{n} u_{n+1}+B_{n} u_{n}+C_{n} u_{n-1}=D f_{n}, \quad 1 \leq n \leq M-1, \\
u_{M}=\overrightarrow{0}, \quad u_{1}=u_{2} .
\end{gathered}
$$

Here, matrices $A_{n}, B_{n}, C_{n}$, and $D$ are the same as in the first example, and

$$
\begin{aligned}
& f_{n}=\left[\begin{array}{c}
f_{n}^{0} \\
f_{n}^{1} \\
f_{n}^{2} \\
\vdots \\
f_{n}^{N}
\end{array}\right]_{(N+1) \times 1}, \\
& f_{n}^{k+1}=f\left(t_{k+1}, x_{n}\right)=[(x+2) \cos x+\sin x+1] \\
& \times\left(e^{t}-1-t\right)+e^{t}(\cos x+1), \quad 1 \leq k \leq N-1,
\end{aligned}
$$

$$
\begin{aligned}
& f_{n}^{0}=\left(1-3 e^{-1}\right)(\cos x+1), \quad 0 \leq n \leq M, \\
& f_{n}^{N}=-e^{-1}(\cos x+1), \quad 0 \leq n \leq M .
\end{aligned}
$$

For the solution of matrix equation (94), we will use modified Gauss elimination method. We seek a solution of the matrix equation by the following form:

$$
u_{n}=\alpha_{n+1} u_{n+1}+\beta_{n+1}, \quad n=M-1, \ldots, 2,1,
$$

where $u_{M}=\overrightarrow{0}, \alpha_{1}$ is identity and $\beta_{1}$ is zero matrices, and

$$
\begin{gathered}
\alpha_{n+1}=-\left(B_{n}+C_{n} \alpha_{n}\right)^{-1} A_{n} \\
\beta_{n+1}=\left(B_{n}+C_{n} \alpha_{n}\right)^{-1}\left(D_{n} \varphi_{n}-C_{n} \beta_{n}\right), \\
n=1,2,3, \ldots, M-1 .
\end{gathered}
$$

Second, for the approximate solutions of nonlocal boundary value problem (92), we use second order of accuracy difference scheme (71) and the formulas

$$
\begin{gathered}
3 u_{0}^{k}-4 u_{1}^{k}+u_{2}^{k}=0, \quad 1 \leq k \leq N-1, \\
10 u_{0}^{k}-15 u_{1}^{k}+6 u_{2}^{k}-u_{3}^{k}=0, \quad 1 \leq k \leq N-1, \\
2 u_{M}^{k}-5 u_{M-1}^{k}+4 u_{M-2}^{k}-u_{M-3}^{k}=0, \quad 1 \leq k \leq N-1, \\
u_{M}^{k}=0, \quad 1 \leq k \leq N-1 .
\end{gathered}
$$

The system can be written in the following matrix form:

$$
\begin{gathered}
P_{n} u_{n+2}+E_{n} u_{n+1}+F_{n} u_{n} \\
+G_{n} u_{n-1}+R_{n} u_{n-2}=D f_{n}, \\
u_{2}-4 u_{1}+3 u_{0}=\overrightarrow{0}, \\
10 u_{0}^{k}-15 u_{1}^{k}+6 u_{2}^{k}-u_{3}^{k}=\overrightarrow{0}, \\
u_{M}=\overrightarrow{0}, \quad 2 u_{M}^{k}-5 u_{M-1}^{k}+4 u_{M-2}^{k}-u_{M-3}^{k}=\overrightarrow{0}, \\
2 \leq n \leq M-2 .
\end{gathered}
$$

Here, $P_{n}$ and $R_{n}$ zero matrices and $E_{n}, F_{n}, G_{n}$, and $D$ are given in the first example, and also $f_{n}$ is given in the first order accuracy difference scheme. For the solution of matrix equation (99), we will use modified Gauss elimination method. We seek a solution of the matrix equation in the following form:

$$
\begin{gathered}
u_{n}=\alpha_{n+1} u_{n+1}+\beta_{n+1} u_{n+2}+\gamma_{n+1}, \quad n=M-2, \ldots, 1, \\
u_{M-1}=\left(I-\frac{4}{5} \alpha_{M-1}+\frac{1}{5} \alpha_{M-2} \alpha_{M-1}\right)^{-1} \\
\times\left(\frac{4}{5} \gamma_{M-1}-\frac{1}{5} \alpha_{M-2} \gamma_{M-1}-\frac{1}{5} \gamma_{M-2}\right) \\
u_{M}=\overrightarrow{0}
\end{gathered}
$$


TABLE 1: Errors for the approximate solution of problem (73).

\begin{tabular}{lccc}
\hline Difference schemes & $N=M=10$ & $N=M=20$ & $N=M=40$ \\
\hline Difference scheme (67) & 0.1983 & 0.0449 & 0.0201 \\
Difference scheme (71) & 0.056 & 0.0014 & 0.0003994 \\
\hline
\end{tabular}

TABLE 2: Errors for the approximate solution of problem (87).

\begin{tabular}{lccc}
\hline Difference schemes & $N=M=10$ & $N=M=20$ & $N=M=40$ \\
\hline Difference scheme (67) & 0.1813 & 0.0516 & 0.0202 \\
Difference scheme (71) & 0.0049 & 0.001 & 0.0002381 \\
\hline
\end{tabular}

TABLE 3: Errors for the approximate solution of problem (94).

\begin{tabular}{lccc}
\hline Difference schemes & $N=M=10$ & $N=M=20$ & $N=M=40$ \\
\hline Difference scheme (67) & 0.1498 & 0.0713 & 0.0347 \\
Difference scheme (71) & 0.0482 & 0.019 & 0.0047 \\
\hline
\end{tabular}

where $\alpha_{j}, \beta_{j}$ are $(N+1) \times(N+1)$ square matrices and $\gamma_{n}(j=$ $1, \ldots, M)$ are $(N+1) \times 1$ column matrices defined by

$$
\begin{gathered}
T_{n}=F_{n}+G_{n} \alpha_{n}+R\left(\beta_{n-1}+\alpha_{n-1} \alpha_{n}\right), \\
\alpha_{n+1}=-\left(T_{n}\right)^{-1}\left(E_{n}+G_{n} \beta_{n}+R \alpha_{n-1} \alpha_{n}\right), \\
\beta_{n+1}=\left(T_{n}\right)^{-1} P_{n}, \\
\gamma_{n+1}=\left(T_{n}\right)^{-1}\left(D f_{n}-G_{n} \gamma_{n}-R_{n}\left(\alpha_{n-1} \gamma_{n}+\gamma_{n-1}\right)\right), \\
\alpha_{1}=\frac{4}{3} I, \quad \beta_{1}=-\frac{1}{3} I, \quad \gamma_{1}=\overrightarrow{0}, \\
\alpha_{2}=\frac{8}{5} I, \quad \beta_{2}=-\frac{3}{5} I, \quad \gamma_{2}=\overrightarrow{0} .
\end{gathered}
$$

Using formulas (100) and (101), we can compute $U_{n}, 1 \leq k \leq$ $M-1$.

Now, let us give the results of numerical analysis. The numerical solutions are recorded for different values of $N=M$, and $u\left(t_{k}, x_{n}\right)$ represents the exact solution, and $u_{n}^{k}$ represents the numerical solution at $\left(t_{k}, x_{n}\right)$. For their comparison, the errors are computed by

$$
E_{M}^{N}=\max _{1 \leq k \leq N-1,1 \leq n \leq M-1}\left|u\left(t_{k}, x_{n}\right)-u_{n}^{k}\right| .
$$

Thus, the results given in Tables 1,2 , and 3 show that the second order of accuracy difference scheme (71) is more accurate comparing with the first order of accuracy difference scheme (67).

\section{Conclusion}

In this paper, we presented first and second order stable difference schemes for solving the second order multidimensional hyperbolic equation with nonlocal integral and Neumann or nonclassical boundary conditions. Stability of the difference schemes do not depend on any additional condition between $h$ and $\tau$. The numerical results given in the previous sections demonstrate the efficiency and good accuracy of these schemes. Finally we would like to mention that this technique can be applied to get the highest order stable difference schemes.

\section{Conflict of Interests}

The authors declare that there is no conflict of interests regarding the publication of this paper.

\section{References}

[1] A. Ashyralyev and P. E. Sobolevskii, New Difference Schemes for Partial Differential Equations, Operator Theory: Advances and Applications, Birkhauser, Basel, Switzerland, 2004.

[2] D. G. Gordeziani and G. A. Avalishvili, "Time-nonlocal problems for schrodinger type equations. I. Problems in abstract spaces," Differential Equations, vol. 41, no. 5, pp. 703-711, 2005.

[3] A. Ashyralyev and O. Gercek, "Nonlocal boundary value problems for elliptic-parabolic differential and difference equations," Discrete Dynamics in Nature and Society, vol. 2008, Article ID 904824, 16 pages, 2008.

[4] A. S. Berdyshev and E. T. Karimov, "Some non-local problems for the parabolic-hyperbolic type equation with noncharacteristic line of changing type," Central European Journal of Mathematics, vol. 4, no. 2, pp. 183-193, 2006.

[5] A. Ashyralyev and O. Yildirim, "On multipoint nonlocal boundary value problems for hyperbolic differential and difference equations," Taiwanese Journal of Mathematics, vol. 14, no. 1, pp. 165-194, 2010.

[6] A. Ashyralyev and N. Aggez, "A note on the difference schemes of the nonlocal boundary value problems for hyperbolic equations," Numerical Functional Analysis and Optimization, vol. 25, no. 5-6, pp. 439-462, 2004.

[7] P. Shi, "Weak solution to evolution problem with a nonlocal constraint," SIAM Journal on Mathematical Analysis, vol. 24, pp. 46-58, 1993.

[8] L. S. Pulkina, "A non-local problem with integral conditions for hyperbolic equations," Electronic Journal of Differential Equations, vol. 1999, pp. 1-6, 1999.

[9] M. Dehghan, "On the solution of an initial-boundary value problem that combines neumann and integral condition for the wave equation," Numerical Methods for Partial Differential Equations, vol. 21, no. 1, pp. 24-40, 2005.

[10] M. Ashyraliyev, "A note on the stability of the integraldifferential equation of the hyperbolic type in a Hilbert space," Numerical Functional Analysis and Optimization, vol. 29, no. 78, pp. 750-769, 2008.

[11] D. Orlovsky and S. Piskarev, “The approximation of BitzadzeSamarsky type inverse problem for elliptic equations with Neumann conditions," Contemporary Analysis and Applied Mathematics, vol. 1, no. 2, pp. 118-131, 2013.

[12] L. S. Pulkina, "On solvability in $L_{2}$ of nonlocal problem with integral conditions for hyperbolic equations," Differentsial'nye Uravneniya, vol. 36, pp. 279-280, 2000.

[13] N. Aggez and A. Ashyralyev, "Finite difference method for hyperbolic equations with the nonlocal integral condition," Discrete Dynamics in Nature and Society, vol. 2011, Article ID 562385, 15 pages, 2011.

[14] H. Soltanov, "A note on the Goursat problem for a multidimensional hyperbolic equation," Contemporary Analysis and Applied Mathematics, vol. 1, no. 2, pp. 98-106, 2013. 
[15] S. Kharibegashvili, "On the solvability of one multidimensional version of the first Darboux problem for some nonlinear wave equations," Nonlinear Analysis, Theory, Methods and Applications, vol. 68, no. 4, pp. 912-924, 2008.

[16] A. I. Kozhanov and L. S. PulKina, "On the solvability of boundary value problems with a nonlocal boundary condition of integral form for multidimensional hyperbolic equations," Differential Equations, vol. 42, no. 9, pp. 1233-1246, 2006.

[17] L. S. Pulkina, "Nonlocal problem with a first-kind integral condition for a multidimensional hyperbolic equation," Doklady Mathematics, vol. 76, no. 2, pp. 741-743, 2007.

[18] A. A. Samarskii, "Local one-dimensional difference schemes for multi-dimesional hyperbolic equations in an arbitrary region," Zhurnal Vychislitel'noi Matematiki i Matematicheskoi, vol. 4, no. 4, pp. 638-648, 1964.

[19] R. K. Mohanty, "Stability interval for explicit difference schemes for multi-dimensional second-order hyperbolic equations with significant first-order space derivative terms," Applied Mathematics and Computation, vol. 190, no. 2, pp. 1683-1690, 2007.

[20] R. L. Higdon, "Absorbing boundary conditions for difference approximations to the multi-dimensional wave equation," Mathematics of Computation, vol. 47, no. 176, pp. 437-459, 1986.

[21] X. Liu, X. Cui, and J. Sun, "FDM for multi-dimensional nonlinear coupled system of parabolic and hyperbolic equations," Journal of Computational and Applied Mathematics, vol. 186, no. 2, pp. 432-449, 2006.

[22] A. Ashyralyev and P. E. Sobolevskii, "A note on the difference schemes for hyperbolic equations," Abstract and Applied Analysis, vol. 6, pp. 63-70, 2001.

[23] P. E. Sobolevskii, Difference Methods for the Approximate Solution of Differential Equations, Voronezh State University Press, Voronezh, Russia, 1975.

[24] A. A. Samarskii and E. S. Nikolaev, Numerical Methods for Grid Equations, Iterative Methods, vol. 2, Birkhauser, Basel, Switzerland, 1989. 


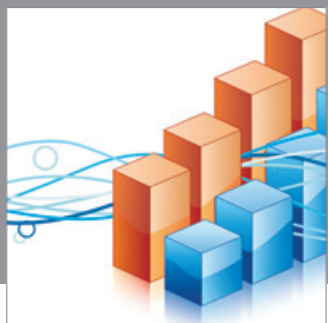

Advances in

Operations Research

mansans

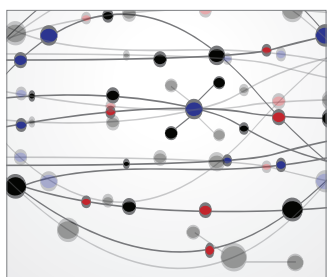

The Scientific World Journal
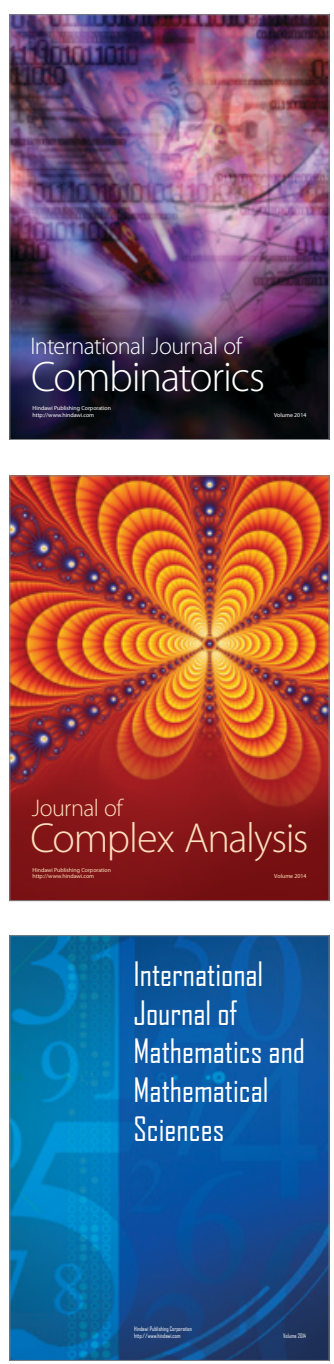
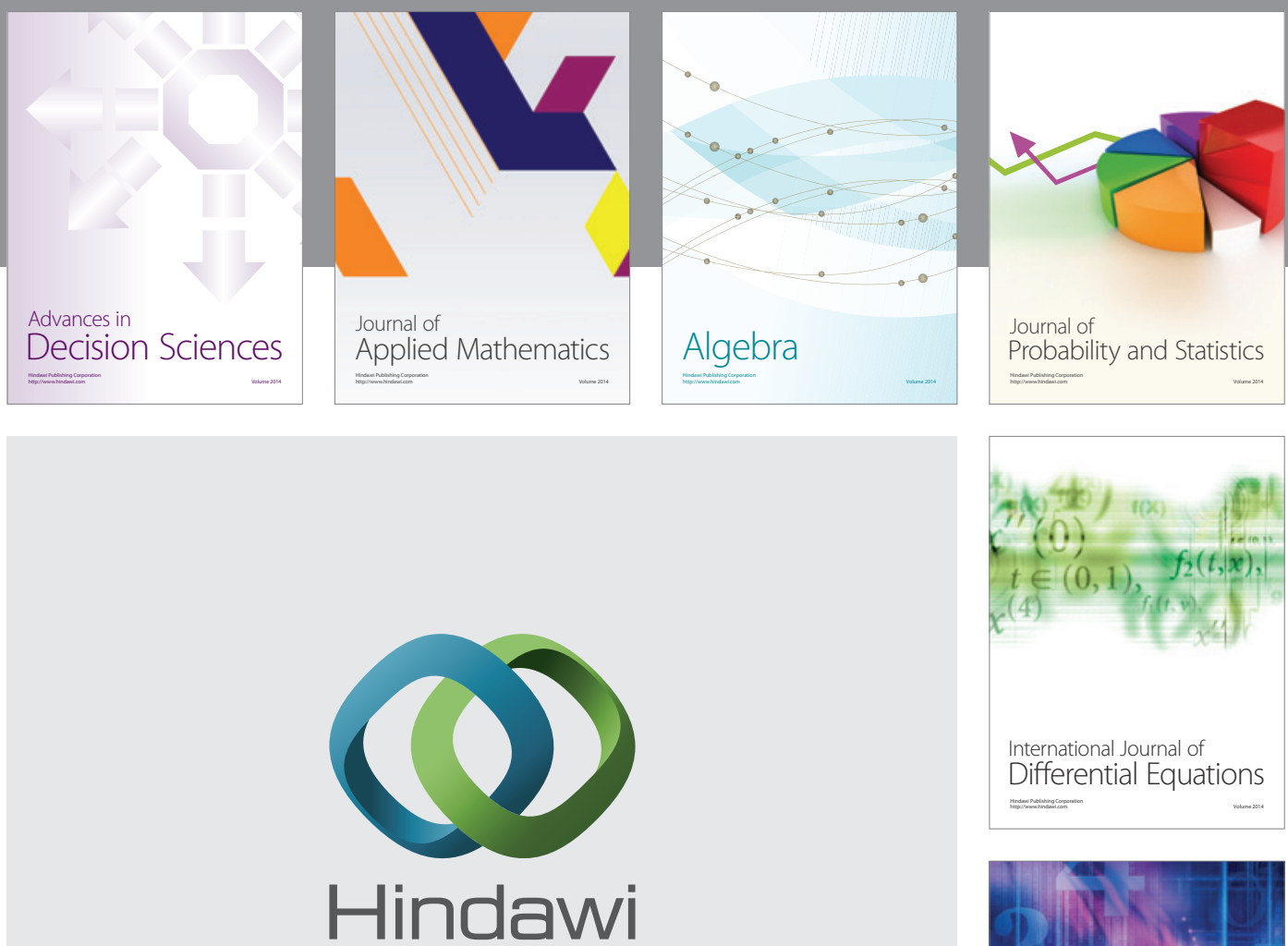

Submit your manuscripts at http://www.hindawi.com
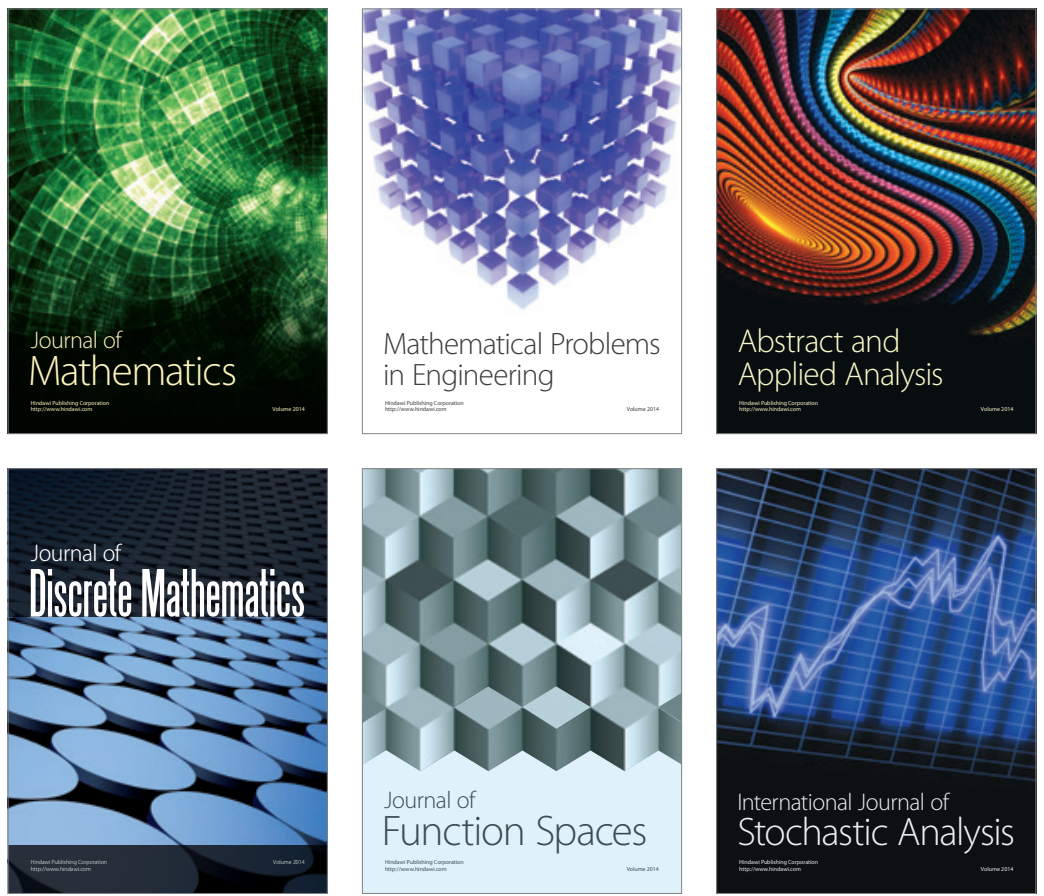

Journal of

Function Spaces

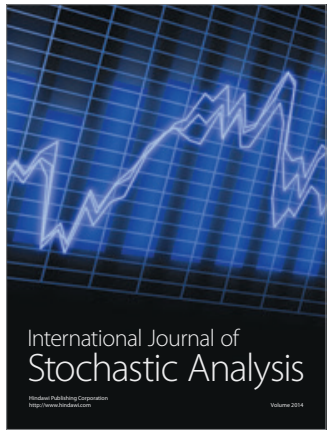

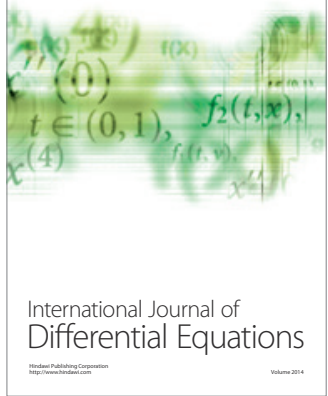
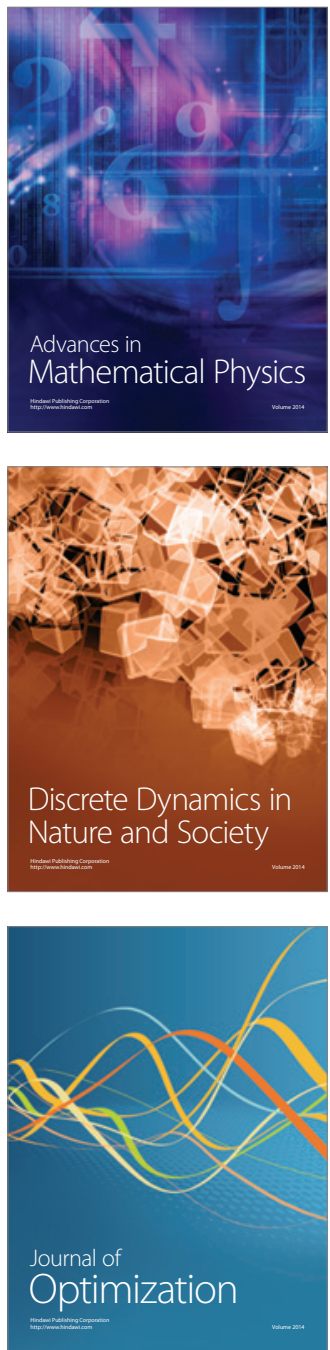